\title{
Interference-Aware Joint Routing and TDMA Link Scheduling for Static Wireless Networks
}

\author{
Yu Wang Weizhao Wang Xiang-Yang Li Wen-Zhan Song
}

\begin{abstract}
We study efficient interference-aware joint routing and TDMA link scheduling for a multihop wireless network to maximize its throughput. Efficient link scheduling can greatly reduce the interference effect of close-by transmissions. Unlike the previous studies that often assume a unit disk graph model, we assume that different terminals could have different transmission ranges and different interference ranges. In our model, it is also possible that a communication link may not exist due to barriers or is not used by a predetermined routing protocol, while the transmission of a node always result interference to all non-intended receivers within its interference range.

Using a mathematical formulation, we develop interference aware joint routing and synchronized TDMA link schedulings that optimize the networking throughput subject to various constraints. Our linear programming formulation will find a flow routing whose achieved throughput is at least a constant fraction of the optimum, and the achieved fairness is also a constant fraction of the requirement. Then, by assuming known link capacities and link traffic loads, we study link scheduling under the RTS/CTS interference model and the protocol interference model with fixed transmission power. For both models, we present both efficient centralized and distributed algorithms that use time slots within a constant factor of the optimum. We also present efficient distributed algorithms whose performances are still comparable with optimum, but with much less communications. We prove that the time-slots needed by our faster distributed algorithms are only at most $O(\min (\log n, \log \psi))$ for RTS/CTS interference model and protocol interference model. Our theoretical results are corroborated by extensive simulation studies.
\end{abstract}

Index Terms - Link scheduling, Interference, Graph Coloring, Distributed Algorithm, Wireless Networks.

\section{INTRODUCTION}

Wireless multi-hop radio networks such as ad hoc, mesh, or sensor networks are formed of autonomous nodes communicating via radio. Wireless networks draw lots of attentions in recent years due to their potential applications in various areas. For example, wireless mesh networks are being used as the last mile for extending the Internet connectivity for mobile nodes. These networks behave almost like wired networks since they have infrequent topology changes, limited node failures, etc.. For wireless mesh networks or sensor networks, the aggregate traffic load of each routing node changes infrequently also. A unique characteristic of wireless networks is that the radio sent out by a wireless terminal will be received by all the terminals within its transmission range, and also possibly causes signal interference to some terminals that are not intended receivers. In other words, the communication channels are shared by the wireless terminals. Thus, one of the major problems facing wireless networks is the

Y. Wang is with the University of North Carolina at Charlotte, USA. Email: yu.wang@uncc.edu. W. Wang is with Google Inc., USA. Email: weizhao@google.com. X.-Y. Li is with Illinois Institute of Technology, USA and Microsoft Research Asia, China, Email: xli@cs.iit.edu. W.-Z. Song is with Washington State University, USA. Email: song@vancouver.wsu.edu. reduction of capacity due to interference caused by simultaneous transmissions. Using multiple channels and multiple radios can alleviate but not eliminate the interference. To achieve robust and collision free communication, there are two alternatives. One is to utilize a random access MAC layer scheme. The other is to carefully construct a transmission schedule. One variant, link scheduling in the context of time division multiplexing (TDM) is the subject of this paper.

In this paper, we assume that the time is slotted and synchronized. A link scheduling is to assign each link a set of time slots $\subset[1, T]$ on which it will transmit, where $T$ is the scheduling period. A link scheduling is interference-aware (or called valid) if a scheduled transmission on a link $x \rightarrow y$ will not result in a collision at either node $x$ or node $y$ (or any other node). In this context, two types of collisions must be avoided, namely, primary interference and secondary interference. Link scheduling has received a great attention from both networking and theory fields [1]-[9] in the past few years due to its application for assigning time slots in TDMA MAC protocols that eliminate collision, guarantee fairness. Many scheduling problems in wireless networks have been shown to be NP-complete, including TDMA broadcast scheduling [10], link scheduling [11], [12]. For some of these problems, even polynomial-time algorithms with constant approximation ratios appear unlikely for general graphs.

Previous studies on link scheduling either assume a very general graph model or assume a very specific graph model such as unit disk graph (UDG). It is widely accepted in the wireless networking community that neither a general graph model nor UDG model accurately captures unique properties of wireless networks. A general graph model could not capture a certain geometry property of wireless networks, e.g., two nodes must be within certain distance to be able to communicate directly (or one node's transmission could interfere the other node's reception). A UDG model is idealistic since in practice two nearby nodes may still be unable to communicate due to various reasons such as barrier and path fading. In this paper, we give efficient centralized and distributed algorithms to obtain a valid link scheduling with theoretically proven performances for a more realistic wireless network model.

For wireless networks, another challenging issue is to route the flow cooperatively among all flows to maximize the network throughput. For example of sensor networks, if routing scheme is not designed carefully, nodes near the sink node will get a large share of the network bandwidth than the nodes that are far away from the sink nodes. Thus, given demands of nodes, we need jointly optimize the routing and TDMA link scheduling to maximize the throughput.

The main contributions of this paper are as follows.

(1) Theoretical Performance Guarantee for Efficient Algorithms: We first consider the joint routing and scheduling problem to maximize either the min-fairness or maximize the 
network throughput under a given min-fairness requirement $\lambda_{0} \geq$ 0 . We present a linear programming formulation based on both necessary and sufficient conditions for schedulable flows under various interference models. Based on this, we design a joint routing and TDMA link scheduling algorithm that will achieve a network throughput within a constant factor of the optimum. Here, we consider two interference models: RTS/CTS model and fixed power protocol interference model (fPrIM). After flow routing is computed, we then present both centralized and distributed link scheduling algorithms that use time slots at most a constant factor of the optimum. All algorithms involve a novel study of interference properties in wireless networks. One of our distributed algorithms has not only small communication complexity, but also good performance guarantee that is only logarithmic of the ratio between the maximum and minimum interference range. Specifically, we prove that the time-slots needed by our faster distributed algorithms are only at $\operatorname{most} O(\min (\log n, \log \psi))$ for RTS/CTS model and fPrIM model, where $\psi$ is the ratio between the largest and smallest interference ranges among all $n$ nodes. Although some of our algorithms are similar to some algorithms proposed before, to the best of our knowledge, we are the first one to prove asymptotic optimal bounds for the performance.

(2) More Realistic Model: We address the link scheduling in a more realistic networking model: (1) each node has its own transmission power and thus its own transmission range; (2) that the receiver must be within the transmission range of the sender is only a necessary (but not sufficient) condition for two nodes to communicate directly, i.e., two nearby nodes may still be unable to communicate directly; (3) if a node $v$ is within certain distance of a sender $u$, then the transmission by $u$ will interfere the reception of node $v$. In summary, the communication graph could be an arbitrary geometry graph. Notice that similar realistic models using weighted and unweighted flows, modeling interference range to be different from transmission range, etc. have all been proposed and modeled in earlier work, e.g. in [4], [7], [13], and heuristic algorithms have been given for each or all of these. Our contributions here are that we provide theoretical bounds for link-scheduling algorithms in these cases.

(3) Both Weighted and Unweighted Flow: In several wireless networks (e.g., mesh, sensor networks), we can estimate the traffic demand by each wireless node. Thus, based on a given routing algorithm, we can predict the average traffic load $f(e)$ on each link $e$ of the network. We then design link scheduling algorithms to meet this traffic demand if possible. We model this by assuming that each link $e$ has an integral weight $w(e)$ specifying the number of slots it needed in a period to support its traffic load. Here $w(e)=\left\lceil T \cdot \frac{f(e)}{\mathbf{c}(e)}\right\rceil$, where $\mathbf{c}(e)$ is the capacity of link $e$ if there is no interference, and $T$ is a given period for a schedule. In certain networks, it is difficult, if not impossible, to estimate the load of every link. We then assume that each node needs one time slot for transmission and our objective is to design a scheduling that minimizes $T$.

The rest of the paper is organized as follows. Section II discusses our network and interference models and formally defines the problem studied in this paper. A mixed integer programming formulation of proposed problems is presented in Section III. Our centralized and distributed algorithms for link scheduling are given in Section IV and Section V, respectively. We also analyze the theoretical guaranteed performances of our algorithms. In Section VI, we study how to assign time slots to links when each link has a requirement of the least number of time slots needed. Our simulation studies are reported in Section VII. In Section VIII, we briefly review the related works in the literature. We conclude our paper in Section IX with the discussion of some possible future works. A preliminary conference version of this article appeared in [14]. Due to space limit, some detailed proofs are omitted in this version with a simple reference to [14].

\section{System Model AND ASSUMPTIONS}

\section{A. Network and Interference Models}

Network Model: We assume that there is a set $V$ of communication terminals deployed in a plane. Each wireless terminal is only equipped with single radio interface. The complete communication graph is a directed graph $G=(V, E)$, where $V=$ $\left\{v_{1}, \ldots, v_{n}\right\}$ is the set of terminals and $E$ is the set of possible directed communication links. Every terminal $v_{i}$ has a transmission range $t_{i}$ such that the necessary condition for a terminal $v_{j}$ to receive correctly the signal from $v_{i}$ is $\left\|v_{i}-v_{j}\right\| \leq t_{i}$, where $\left\|v_{i}-v_{j}\right\|$ (sometimes we denote it as $d_{i, j}$ for simplicity) is the Euclidean distance between $v_{i}$ and $v_{j}$. Notice that $\left\|v_{i}-v_{j}\right\| \leq t_{i}$ is not the sufficient condition for $\left(v_{i}, v_{j}\right) \in E$. Some links do not belong to $G$ because of either the physical barriers or the selection of routing protocols. This is the major distinction of our model with the majority previous studies on link scheduling. To the best of our knowledge, only [7] used the similar model as ours. We always use $\mathbf{L}_{i, j}$ to denote $\left(v_{i}, v_{j}\right)$ hereafter. For a link $e$, we use $\mathbf{c}(e)$ to denote its expected capacity when no interference links are transmitting simultaneously. Each terminal $v_{i}$ also has an interference range $r_{i}$ such that $v_{j}$ is interfered by the signal from $v_{i}$ if $\left\|v_{i}-v_{j}\right\| \leq r_{i}$ and $v_{j}$ is not the intended receiver. The interference range $r_{i}$ is not necessarily same as the transmission range $t_{i}$. Typically, $r_{i}>t_{i}$. We call the ratio between them as the Interference-Transmission Ratio for node $v_{i}$, denoted as $\gamma_{i}=\frac{r_{i}}{t_{i}}$. In practice, $2 \leq \gamma_{i} \leq 4$. For all wireless nodes, let $\gamma=\max _{v_{i} \in V} \frac{r_{i}}{t_{i}}$. For a node $u$, we use $\Lambda^{+}(u)$ to denote the set of incoming links (all directed links pointed to $u$ ). Similarly, we use $\Lambda^{-}(u)$ to denote the set of outgoing links at node $u$.

INTERFERENCE Models: To schedule two links at the same time slot, we must ensure that the schedule will avoid the interference. Two different types of interference have been studied in the literature, namely, primary interference and secondary interference. Primary interference occurs when a node transmits and receives packets at the same time. Secondary interference occurs when a node receives two or more separate transmissions. Here all transmissions could be intended for this node, or only one transmission is intended for this node (thus, all other transmissions are interference to this node). In addition to these interferences, there could have some other constraints on the scheduling, e.g., the radio networks that deploy the IEEE 802.11 protocol with requestto-send and clear-to-send (RTS/CTS) mechanism will pose some additional constraints. Several different interference models have been used to model the interferences in wireless networks. We briefly review the models we use in this paper.

Protocol Interferences Model (PrIM) [15]: In this model, a transmission by a node $v_{i}$ is successfully received by a node $v_{j}$ iff the intended destination $v_{j}$ is sufficiently apart from the source of any other simultaneous transmission, i.e., $\left\|v_{k}-v_{j}\right\| \geq$ $(1+\eta)\left\|v_{i}-v_{j}\right\|$ for any node $v_{k} \neq v_{i}$. Here constant $\eta>0$ models situations where a guard zone is specified by the protocol 
to prevent a neighboring node from transmitting on the same channel at the same time. This model implicitly assumed that each node $v_{k}$ will adopt the power control mechanism when it transmits signals. Simulation analysis [16] as well as the analytical results [17] indicate that the PrIM does not necessarily provide a comprehensive view of reality due to the aggregate effect of interference in wireless networks. However, it does provide some good estimations of interference and most importantly it enables a theoretical performance analysis of a number of protocols designed in the literature. Link scheduling under PrIM and network model similar to ours has been studied in [7].

Fixed Power Protocol Interferences Model (fPrIM): We adopt the following interference model throughout this paper. We assume that a node will not dynamically change its power based on the intended receiver in a packet-level. Note that this assumption does not preclude the power control that can further reduce the power consumption. We only assume that there is no power adaptation at the packet level and the power is not adjustable for a certain period of time, which is close to the real situation. However, we do assume that each node $v_{i}$ has its own fixed transmission power and thus a fixed transmission range $t_{i}$. We also assume that each node $v_{k}$ has an interference range $r_{k}$ such that any node $v_{j}$ will be interfered by the signal from $v_{k}$ if $\left\|v_{k}-v_{j}\right\| \leq r_{k}$ and node $v_{k}$ is sending signal to some node other than $v_{j}$. In other words, the transmission from $v_{i}$ to $v_{j}$ is viewed successful if $\left\|v_{k}-v_{j}\right\|>r_{k}$ for every node $v_{k}$ transmitting in the same time slot using the same channel.

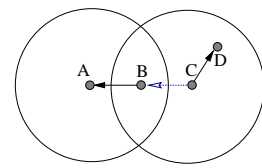

(a) Due to RTS

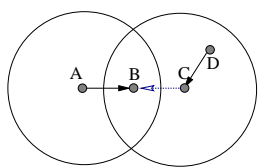

(b) Due to CTS
Fig. 1. Communication Restriction by RTS/CTS.

RTS/CTS Model: This model was also studied previously, e.g., [1]. For every pair of transmitter and receiver, all nodes that are within the interference range of either the transmitter or the receiver cannot transmit. Figure 1(a) shows the case that communication from $B$ to $A$ and $C$ to $D$ cannot take place simultaneously due to RTS. Figure 1(b) shows the case that communication from $A$ to $B$ and $D$ to $C$ cannot take place simultaneously due to CTS. Although RTS/CTS is not the interference itself, for convenience of our notation, we will treat the communication restriction due to RTS/CTS as RTS/CTS interference model. Thus, for every pair of simultaneous communication links, say $v_{i} v_{j}$ and $v_{p} v_{q}$, it should satisfy that (1) they are distinct four nodes, i.e., $v_{i} \neq v_{j} \neq v_{p} \neq v_{q}$; (2) $v_{i}$ and $v_{j}$ are not in the interference ranges of $v_{p}$ and $v_{q}$, and vice versa. Figure 2(a) shows an example where link $\mathbf{L}_{i, j}$ interferes $\mathbf{L}_{p, q}$. Here, a solid circle with center $v$ denotes the transmission region and dotted circle denotes the interference region of node $v$. The interference region, denoted by $I_{i, j}$, of a link $\mathbf{L}_{i, j}$ is the union of the interference region of nodes $v_{i}$ and $v_{j}$. See Figure 2(b) for illustration. When a directed link $v_{i} v_{j}$ (or $\left.v_{j} v_{i}\right)$ is active, all simultaneous transmitting links $v_{p} v_{q}$ cannot have an end-point inside the area $I_{i, j}$.

There are also other interference models, e.g., Transmitter Interference Model [18] and Physical Interference Model. However, in this paper, we mainly focus on joint routing and link scheduling for fPrIM and RTS/CTS models. Note that these two models are different, e.g., in Figure 1(a), links $B A$ and $C D$ can be assigned the same channel in fPrIM model, but not in RTS/CTS model. Similar statement holds for links $A B$ and $D C$ in Figure 1(b).

Assume that the communication links in the wireless network are predetermined. Given a communication graph $G=(V, E)$, we use the conflict graph (e.g., [13]) $F_{G}$ to represent the interference in $G$. Each vertex (denoted by $\mathbf{L}_{i, j}$ ) of $F_{G}$ corresponds to a directed link $\left(v_{i}, v_{j}\right)$ in the communication graph $G$. There is an edge between vertex $\mathbf{L}_{i, j}$ and vertex $\mathbf{L}_{p, q}$ in $F_{G}$ if and only if $\mathbf{L}_{i, j}$ conflicts with $\mathbf{L}_{p, q}$ due to interference. Recall that whether two links conflict depends on the interference model used underneath, e.g., fPrIM model or RTS/CTS model. Thus, for a given communication graph $G$, the interference graph $F_{G}$ may be different. To avoid the confusion, we use $F_{G}^{P}$ to denote the interference graph under the fPrIM model and $F_{G}^{D 2}$ to denote interference graph under RTS/CTS model.

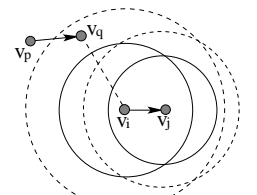

(a) $\mathbf{L}_{i, j}$ interferes $\mathbf{L}_{p, q}$

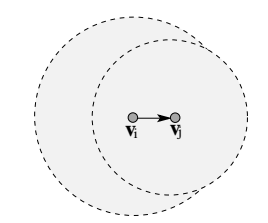

(b) Interference region $I_{i, j}$
Fig. 2. RTS/CTS Interference Model.

\section{B. Problem Formulation}

Assume that each ordinary node $u$ will aggregate the traffic from all its users and then route them to the Internet through some gateway nodes. We use $\ell_{O}(u)$ to denote the total aggregated outgoing traffic of node $u$ users and $\ell_{I}(u)$ to denote the total aggregated incoming traffic of node $u$ users. We will mainly concentrate on incoming traffic in this paper. For notation simplicity, we use $\ell(u)$ to denote such load for node $u$. Notice that the traffic $\ell(u)$ is not requested to be routed through a specific gateway node, neither requested to be using a single routing path. We also assume that among the set $V$ of all wireless nodes, some of them have gateway functionality and provides the connectivity to the Internet. For simplicity, let $\mathcal{S}=\left\{\mathbf{s}_{1}, \mathbf{s}_{2}, \cdots, \mathbf{s}_{g}\right\}$ be the set of $g$ gateway nodes, where $\mathbf{s}_{i}$ is actually node $v_{n+i-g}$. All other wireless nodes $v_{i}$ (for $1 \leq i \leq n-g$ ) are called ordinary wireless nodes. We assume that the gateway nodes will not act as relay node for a pair of ordinary wireless nodes. The routing problem is to decide a multi-path routing structure for each source node and an assignment of its flow to all links in the network. The flow assignment should satisfy certain restrictions such as flow conservation. Most importantly, the assigned flow should be schedulable by the coupled link scheduling method.

After the flow is assigned to each link, we then need to decide when a node should be actively sending data to a neighboring node, when TDMA link scheduling is adopted. Our objective of the scheduling problem is to give each link $\mathbf{L} \in G$ a transmission schedule $\mathcal{S}(\mathbf{L})$, which is the list of time slots it could send packets such that the schedule is interference-free and the overall throughout of the network is maximized. Let $X_{e, t} \in\{0,1\}$ be the indicator variable which is 1 iff $e$ will transmit at time $t$. We will focus on periodic schedules in this paper. A schedule is periodic with period $T$ if, for every link $e$ and time slot $t, X_{e, t}=X_{e, t+i \cdot T}$ for any integer $i$. For a link $e$, let $I(e)$ denote the set of links $e^{\prime}$ that will cause interference if $e$ and $e^{\prime}$ are scheduled at the same time slot. A schedule $\mathcal{S}$ is interference-free if $X_{e, t}+X_{e^{\prime}, t} \leq 1$ for any $e^{\prime} \in I(e)$. In the graph theory terminology, the interference 
free link scheduling problem is essentially the weighted vertex coloring of $F_{G}$.

When the traffic load of links are unknown, the objective of link scheduling is to find a scheduling with the minimum period. If we schedule all links within a period $\chi$ such that no two links in same time slot interfere with each other, then at least one packet can be delivered over each communication link in every $\chi$ time slots. Thus, $1 / \chi$ is often used to estimate the throughput of the network based on this schedule. The second case is that the average traffic load $f(e)$ of each link is known in advance from the routing. We model this by assuming that each communication link $e$ (vertex in the conflict graph) has a weight $w(e)$ specifying the minimum number of time slots it required in each period. Here $w(e)=\left\lceil T \cdot \frac{f(e)}{\mathbf{c}(e)}\right\rceil$, where $\mathbf{c}(e)$ is the capacity of link $e$ if there is no interference, and $T$ is a given period for a schedule. Our main focus in this paper is how to schedule the communication links in an interference-free manner such that the throughput of the network is maximized, i.e., with the smallest $T$.

Notice that for simplicity we assume that there is only a singlechannel in the network. All our results can be easily extended to the case when multiple channels are available as in [1]. If nodes has a pre-assigned channels for each link, then the link scheduling with multiple channels is just the simple union of a set of schedulings, where each scheduling is for all links using the same channel. However, we agree that the static assignment of correct channels to appropriate links is a bigger factor in determining the performance. If links can dynamically switch channels, then our greedy algorithms will find the channel with the smallest available time slot for each link to be scheduled and the same performances hold.

\section{JOINT Routing AND LINK SCHEDULING}

In this section, we first give a mixed Integer Programming formulation of the problem to be studied.

First assume that each source node has a demand for data rate $\ell(u)$. We want to find a routing that maximizes the minimum fairness, which is defined as the ratio of the achieved data rate over the required data rate. Given a link $e$, let $f(e)$ be the total flow assigned to link $e$. We formulate the max-min-fairness routing problem as follows.

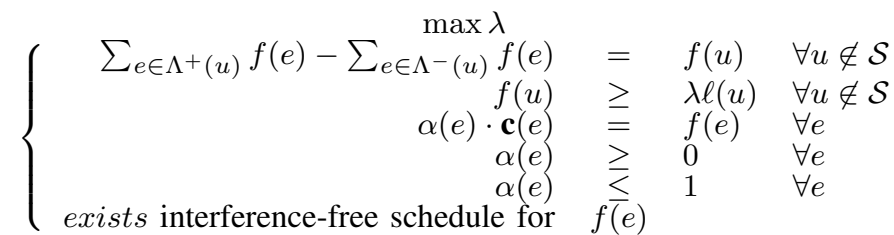

Here $f(u)$ is the achieved data rate for node $u$ with flow assignment $f ; 0 \leq \alpha(e) \leq 1$ is the fraction of the time link $e$ will be actively transmitting to achieve such flow assignment. Notice that, for links that interfere with each other, clearly, the summation of their $\alpha(e)$ should be no more than 1. It is widely known that it is NP-hard to decide whether a feasible scheduling $X_{e, t}$ exists when given the flow $f(e)$ (or equivalently, $\alpha(e)$ ) for wireless networks with interference constraints. Similarly, we can formulate the problem of routing for maximizing the throughput where the objective function is $\max _{u \in V} f(u)$ and the $\lambda$ in the section inequality is replaced by some minimum fairness requirement constant $\lambda_{0} \geq 0$.

Schedulable Flows: We then mathematically formulate the necessary and sufficient condition for schedulable flow $f(e)=$ $\alpha(e) \cdot \mathbf{c}(e)$ : flow $f$ (equivalently, whether a given vector $\alpha(e)$ for all $e$ is schedulable) is schedulable if and only if we can find integer solution $X_{e, t}$ satisfying the following conditions.

$$
\left\{\begin{array}{rlrl}
X_{e, t}+X_{e^{\prime}, t} & \leq 1 & \forall e^{\prime} \in \mathbf{I}(e), \forall e, \forall t, \\
\frac{\sum_{1 \leq t \leq T} X_{e, t}}{T}=\alpha(e) & \forall e, \\
X_{e, t} & \in\{0,1\} \quad \forall e, \forall t,
\end{array}\right.
$$

Recall that here $X_{e, t}$ denotes whether link $e$ is active at time $t \in[1, T]$. For some interference models several papers gave relaxed necessary conditions and relaxed sufficient conditions for schedulable flows that can be decided in polynomial time. For example, for RTS/CTS model with uniform transmission range and uniform interference range, [1] gave a sufficient condition $\alpha(e)+\sum_{e^{\prime} \in \mathbf{I}(e)} \alpha\left(e^{\prime}\right) \leq 1$, and a necessary condition $\alpha(e)+$ $\sum_{e^{\prime} \in \mathbf{I}(e)} \alpha\left(e^{\prime}\right) \leq C(q)$. Here $C(q)$ is a constant depending on the ratio of interference range over the transmission range.

For each of the interference models discussed in this paper, we later will present a necessary and a sufficient condition for schedulable flows. Generally, we have the following theorem (whose proof is deferred to later section)

Theorem 1: Assume that the network is single-channel network. A sufficient condition for a flow defined by $\alpha(e)$ to be schedulable is,

$$
\alpha(e)+\sum_{e^{\prime} \in \mathbf{I}_{\mathcal{M}}(e)} \alpha\left(e^{\prime}\right) \leq 1
$$

and a necessary condition for such flow to be schedulable is,

$$
\alpha(e)+\sum_{e^{\prime} \in \mathbf{I}_{\mathcal{M}}(e)} \alpha\left(e^{\prime}\right) \leq C_{\mathcal{M}} .
$$

Here $\mathbf{I}_{\mathcal{M}}(e) \subseteq \mathbf{I}(e)$ is defined based on the specific interference model $\mathcal{M}$ for the purpose of link scheduling; $C_{\mathcal{M}}$ is a constant depending on the specific interference model and $\gamma . C_{R T S / C T S}$ is a constant defined in Lemma 6; while $C_{f \operatorname{PrIM}}=\left\lceil\frac{2 \pi}{\arcsin \frac{\gamma-1}{2 \gamma}}\right\rceil$ is proved in Lemma 9.

Consequently, we need to solve the following Linear Programming (LP-Flow-fairness) for $\alpha(e)$ such that

$$
\left\{\begin{array}{rll}
\max \lambda & & \\
\sum_{e \in \Lambda^{+}(u)} f(e)-\sum_{e \in \Lambda^{-}(u)} f(e) & =f(u) & \forall u \notin \mathcal{S} \\
f(u) & \geq \lambda \ell(u) & \forall u \notin \mathcal{S} \\
\alpha(e) \cdot \mathbf{c}(e) & =f(e) & \forall e \\
\alpha(e) & \geq 0 & \forall e \\
\alpha(e) & \leq 1 & \forall e \\
\alpha(e)+\sum_{e^{\prime} \in \mathbf{I}_{\mathcal{M}}(e)} \alpha\left(e^{\prime}\right) & \leq 1 & \forall e
\end{array}\right.
$$

In majority applications, we not only have to guarantee certain fairness of the achieved flows for all end wireless devices, but also have to achieve the largest possible throughput under certain fairness constraints. Assume that we have a minimum fairness constraints $\lambda_{0}$. To approximately find the maximum throughput routing, we will solve the following linear programming (LPFlow-throughput) for $\alpha(e)$ such that

$$
\left\{\begin{array}{rlrl}
\max \sum_{i=1}^{g} f\left(\mathbf{s}_{i}\right) & & \\
\sum_{e \in \Lambda^{+}(u)} f(e)-\sum_{e \in \Lambda^{-}(u)} f(e) & =f(u) & & \forall u \notin \mathcal{S} \\
f(u) & \geq \lambda_{0} \ell(u) & \forall u \notin \mathcal{S} \\
\sum_{e \in \Lambda^{-}\left(\mathbf{s}_{i}\right)} f(e)-\sum_{e \in \Lambda^{+}\left(\mathbf{s}_{i}\right)} f(e) & =f\left(\mathbf{s}_{i}\right) & \forall \mathbf{s}_{i} \in \mathcal{S} \\
\alpha(e) \cdot \mathbf{c}(e) & =f(e) & \forall e \\
\alpha(e) & \geq 0 & \forall e \\
\alpha(e) & \leq 1 & \forall e \\
\alpha(e)+\sum_{e^{\prime} \in \mathbf{I}_{\mathcal{M}}(e)} \alpha\left(e^{\prime}\right) & \leq 1 & \forall e
\end{array}\right.
$$

Based on the above linear programming formulations, we will solve $\alpha(e)$ for all links $e$. In following sections, we will present 
both centralized algorithms (Algorithms 1 and 2 for link scheduling in RTS/CTS and fPrIM models respectively) and distributed algorithms for scheduling link activities to achieve the flows. This efficient algorithms, together with our linear programming formulations imply the following theorems.

Theorem 2: Algorithms 1 and 2 together with Algorithm 6 and the linear programming formulation LP-Flow-fairness, produce a feasible interference-free link-channel scheduling whose achieved fairness is at least $\frac{1}{C_{\mathcal{M}}}$ of the optimum.

Proof: Consider an optimum flow assignment defined by $\alpha^{*}(e)$, i.e., the flow supported by a link $e$ is $\alpha^{*}(e) \cdot \mathbf{c}(e)$. From Theorem 1, we know that

$$
\alpha^{*}(e)+\sum_{e^{\prime} \in \mathbf{I}_{\mathcal{M}}(e)} \alpha^{*}(e) \leq C_{\mathcal{M}}
$$

Define a new flow $\alpha^{\prime}$ as $\alpha^{\prime}(e)=\frac{\alpha^{*}(e)}{C_{\mathcal{M}}}$. Obviously,

$$
\alpha^{\prime}(e)+\sum_{e^{\prime} \in \mathbf{I}_{\mathcal{M}}(e)} \alpha^{\prime}(e) \leq 1 .
$$

It is easy to show that the new flow $\alpha^{\prime}$ satisfies all conditions of our linear programming LP-Flow-fairness. In other words, $\alpha^{\prime}$ is a feasible solution for this LP. Consequently, the solution of LPFlow-fairness is at least that of $\alpha^{\prime}$, which is $\frac{1}{C_{\mathcal{M}}}$ of the optimum. This finishes the proof.

Similarly, we have

Theorem 3: Algorithms 1 and 2 together with Algorithm 6 and the linear programming formulation LP-Flow-throughput, produce a feasible interference-free link-channel scheduling whose achieved throughput is at least $\frac{1}{C_{\mathcal{M}}}$ of the optimum, whose achieved fairness is at least $\frac{1}{C_{\mathcal{M}}} \lambda_{0}$.

\section{Centralized Link Scheduling}

In this section, we propose centralized link scheduling algorithms under different interference models when the objective is to schedule every link once and minimize the time-period $T$ used. Some fundamental studies of interference graph here will form the bases for scheduling links when each link has a requirement on the number of time-slots it needed in a scheduling period.

\section{A. Scheduling under RTS/CTS Model}

A number of centralized algorithms for link scheduling have been proposed in the literature, e.g., [1], [7]. A common approach is to assign each link the best possible channels (smallest time slots here) by greedy. The difference between them is the processing order of links: [7] processes links with smaller lengths first while [1] processes links in an arbitrary order (since it uses UDG graph models for both communication and interference). Our centralized algorithm (Algorithm 1) processes links in a special order as in [19]. The basic idea is to first sort links as follows: every time we pick a link, say $\mathbf{L}$, from the remaining graph that has the smallest number of interfered links in the remaining graph and then remove $\mathbf{L}$ from this graph; repeat this till the graph becomes empty. We then assign time slots to links in the reverse order of picked links using the smallest time slot available (not used by interfering links). In summary, a link $e$ with larger $I(e)$ will be more likely processed earlier.

We first present some necessary definitions and properties needed to prove the performance of our algorithms. Given a communication link $\mathbf{L}_{i, j}$, we define the interference radius of link $\mathbf{L}_{i, j}$ as $r_{i, j}=\max \left\{r_{i}, r_{j}\right\}$. If $r_{i}>r_{j}$ or $r_{i}=r_{j}$ and ID

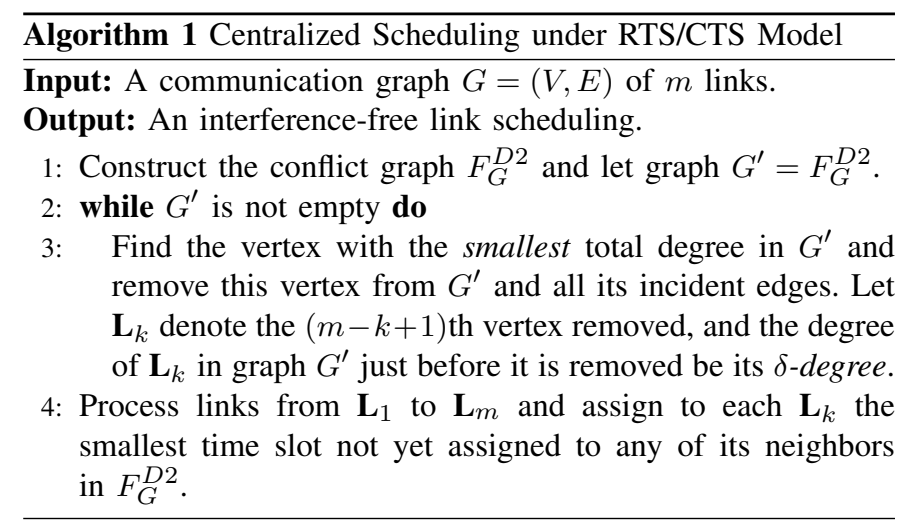

of node $v_{i}$ is larger than the ID of node $v_{j}$, then $v_{i}$ is called the head (denoted as $\left.h_{i, j}\right)$ of link $\left(v_{i}, v_{j}\right)$ and $v_{j}$ is the tail (denoted as $t_{i, j}$ ) of this link. Notice that here, the head of a link is not necessarily the sender of the directed communication link. Given a node $v_{k}$, we use $R\left(v_{k}, x\right)$ to denote the disk centered at $v_{k}$ and with radius $x \cdot r_{k}$. A node $v_{k}$ interferes a node $v_{i}$ if node $v_{i}$ is inside the interference region (i.e., disk $R\left(v_{k}, 1\right)$ ) of node $v_{k}$. We say a link $\mathbf{L}_{p, q}$ interferes a node $v_{k}$ if either $v_{p}$ or $v_{q}$ interferes $v_{k}$. For a given node $v_{k}$, we use $N^{\geq}\left(v_{k}, \alpha\right)$ to denote the set of nodes satisfying that (1) each of their interference radius is at least $r_{k}$; (2) each of them interferes some nodes in $R\left(v_{k}, \alpha\right)$. Notice that a node from $N^{\geq}\left(v_{k}, \alpha\right)$ could be arbitrarily far away from node $v_{k}$. Similarly, for a link $\mathbf{L}_{i, j}$, let $R\left(\mathbf{L}_{i, j}, x\right)$ denote the union of two disks centered at $v_{i}$ and $v_{j}$ respectively with radius $x \cdot r_{i}$ and $x \cdot r_{j}$ respectively. Let $N^{\geq}\left(\mathbf{L}_{i, j}, \alpha\right)$ denote the union of node sets $N^{\geq}\left(v_{i}, \alpha\right)$ and $N^{\geq}\left(v_{j}, \alpha\right)$. The following theorem estimates the local chromatic number based on node degree.

Theorem 4: For a given node $v_{k}$ and any node set $V_{k} \subseteq$ $N^{\geq}\left(v_{k}, \alpha\right)$ with constant $\alpha$, there exists a subset $V_{k}^{\prime}$ of $V_{k}$ with cardinality $\left|V_{k}\right| / C_{\alpha}$ such that each node interferes with each other, where $C_{\alpha} \leq(6 \alpha+1)^{2}+11$.

Proof: We consider a partition of $V_{k}$ : the nodes in and outside region $R\left(v_{k}, 3 \alpha\right)$, denoted by $V_{k}^{1}$ and $V_{k}^{2}$ respectively.

First, we consider the node set $V_{k}^{1}$. Using a simple area argument, there are at most $\frac{\pi\left(\left(3 \alpha+\frac{1}{2}\right) r_{k}\right)^{2}}{\pi\left(\frac{1}{2} r_{k}\right)^{2}}=(6 \alpha+1)^{2}$ disks with radius $\frac{r_{k}}{2}$ can be placed inside the disk $R\left(v_{k}, 3 \alpha\right)$. Thus, there exists a node set in $V_{k}^{1}$ with size at least $\left|V_{k}^{1}\right| /(6 \alpha+1)^{2}$ such that each node in the set interferes with each other.

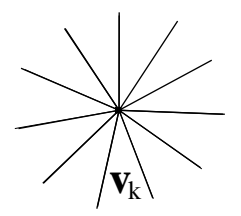

(a) Divide into 11 cones

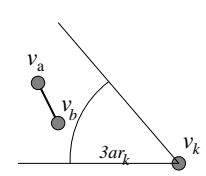

(b) 2 nodes interfere in same cone
Fig. 3. Illustration of the partition of the region.

Second, we consider the node set $V_{k}^{2}$. We divide the whole space into 11 equal cones using 11 rays from $v_{k}$ as shown Figure 3(a). If $v_{a}$ and $v_{b}$ are in the same cone, then $\angle v_{a} v_{k} v_{b}<33^{\circ}$. Let $d_{a, b}=\left\|v_{a}-v_{b}\right\|$. Since $v_{a} \in N^{\geq}\left(v_{k}, \alpha\right), v_{a}$ interfere with some nodes in $R\left(v_{k}, \alpha\right), d_{a, k} \leq r_{a}+\alpha \cdot r_{k}$. Similarly, $d_{b, k} \leq r_{b}+\alpha \cdot r_{k}$. Thus, $\max \left\{d_{a, k}, d_{b, k}\right\} \leq \max \left\{r_{a}, r_{b}\right\}+\alpha \cdot r_{k}$. On the other hand, since both $v_{a}$ and $v_{b}$ are outside $R\left(v_{k}, 3 \alpha\right), \min \left\{d_{a, k}, d_{b, k}\right\} \geq$ 
$3 \alpha \cdot r_{k}$. As shown in Figure $3(\mathrm{~b})$, for $v_{a}$ and $v_{b}$,

$$
\begin{aligned}
d_{a, b}^{2}< & d_{a, k}^{2}+d_{b, k}^{2}-2 \cos \left(33^{\circ}\right) \cdot d_{a, k} \cdot d_{b, k} \\
= & \max \left\{d_{a, k}, d_{b, k}\right\}^{2}+\min \left\{d_{a, k}, d_{b, k}\right\}^{2}- \\
& \frac{5}{3} \max \left\{d_{a, k}, d_{b, k}\right\} \cdot \min \left\{d_{a, k}, d_{b, k}\right\} \\
\leq & \max \left\{d_{a, k}, d_{b, k}\right\}\left[\max \left\{d_{a, k}, d_{b, k}\right\}-\frac{2}{3} \min \left\{d_{a, k}, d_{b, k}\right\}\right] \\
\leq & \left(\max \left\{r_{a}, r_{b}\right\}+\alpha \cdot r_{k}\right) \cdot\left[\max \left\{r_{a}, r_{b}\right\}+\alpha \cdot r_{k}-2 \alpha \cdot r_{k}\right] \\
\leq & \max \left\{r_{a}, r_{b}\right\}^{2}-\alpha^{2} \cdot r_{k}^{2}<\max \left\{r_{a}, r_{b}\right\}^{2} .
\end{aligned}
$$

The transition between the second and third inequalities is because $\max \left\{d_{a, k}, d_{b, k}\right\} \leq \max \left\{r_{a}, r_{b}\right\}+\alpha \cdot r_{k}$ and $\min \left\{d_{a, k}, d_{b, k}\right\} \geq$ $3 \alpha \cdot r_{k}$. Thus, $v_{a}$ interferes with $v_{b}$. Therefore, each pair of nodes in the same cone interfere with each other. This proves that there exists a node set in $V_{k}^{2}$ with size at least $\left|V_{k}^{2}\right| / 11$ such that the nodes in the set interfere with each other.

Consequently, there exists a node set with size at least

$$
\max \left\{\left|V_{k}^{1}\right| /(6 \alpha+1)^{2},\left|V_{k}^{2}\right| / 11\right\} \geq \frac{\left|V_{k}^{1}\right|+\left|V_{k}^{2}\right|}{(6 \alpha+1)^{2}+11}=\frac{\left|V_{k}\right|}{C_{\alpha}}
$$

such that all nodes in the set interfere with each other. Here, $C_{\alpha} \leq(6 \alpha+1)^{2}+11$, and we call it the $\alpha$-hop interference number. Notice that $(6 \alpha+1)^{2}+11$ is an upper bound on $C_{\alpha}$ and it can be improved by using a more tight analysis.

Notice that Theorem 4 works for the interference on nodes only. For a link $e=\mathbf{L}_{i, j}$, let $I^{\geq}(e)$ be the links $e^{\prime}$ interfering with $e$ under RTS/CTS model and whose radius is not smaller than $e$. Following theorem shows a counterpart that works for links also.

Theorem 5: For a given link $e=\mathbf{L}_{i, j}$, at least $\left|I^{\geq}(e)\right| /\left(2 C_{1}\right)$ time slots are needed to schedule all links in $I^{\geq}(e)$.

Proof: For each link $\mathbf{L}_{p, q} \in I^{\geq}(e)$, without loss of generality, we assume that $r_{p} \geq r_{q}$. Recall that $e^{\prime}=\mathbf{L}_{p, q}$ and $e$ interfere by definition. Following we discuss by cases.

Case 1: The interference region of $v_{p}$ covers either $v_{i}$ or $v_{j}$.

Case 2: The interference region of node $v_{p}$ can neither cover $v_{i}$ nor $v_{j}$, and $v_{q}$ is outside the union $R\left(\mathbf{L}_{i j}, 1\right)$ of interference region of $v_{i}$ and $v_{j}$. Clearly, in this case $v_{p}$ must also be outside of $R\left(\mathbf{L}_{i j}, 1\right)$. Since $e$ and $e^{\prime}$ interfere, it must be that the interference region of $v_{q}$ covers either $v_{i}$ or $v_{j}$.

Case 3: The interference region of node $v_{p}$ can neither cover $v_{i}$ nor $v_{j}$, and $v_{q}$ is inside the union $R\left(\mathbf{L}_{i j}, 1\right)$ of interference region of $v_{i}$ and $v_{j}$. Then $v_{p}$ will "interfere" a dummy node $v_{q}$.

In summary, we conclude that at least one end node of $\mathbf{L}_{p, q}$ interferes with some nodes in region $R\left(\mathbf{L}_{i, j}, 1\right)$, i.e., the head of $\mathbf{L}_{p, q}$ is in $N^{\geq}\left(\mathbf{L}_{i, j}, 1\right)$. Recall that $N^{\geq}\left(\mathbf{L}_{i, j}, 1\right)=$ $N^{\geq}\left(v_{i}, 1\right) \bigcup N^{\geq}\left(v_{j}, 1\right)$. The head of $\mathbf{L}_{p, q}$ is either in $N^{\geq}\left(v_{i}, 1\right)$ or $N^{\geq}\left(v_{j}, 1\right)$. Without loss of generality, we assume that at least $\left|I^{\geq}(e)\right| / 2$ heads of the links in $I^{\geq}(e)$ are in $N^{\geq}\left(v_{i}, 1\right)$. From Theorem 4 , there are at least $\left|I^{\geq}(e)\right| /\left(2 C_{1}\right)$ heads that interfere with each other. Thus, there are at least $\left|I^{\geq}(e)\right| /\left(2 C_{1}\right)$ links in $I^{\geq}(e)$ that interfere with each other. This finishes the proof.

Consequently, we have the following necessary condition for any interference-free link scheduling under RTS/CTS model:

Lemma 6: For any time slot $\tau$, any valid RTS/CTS interference-free link scheduling $\mathcal{S}$ must satisfy that

$$
X_{e, \tau}+\sum_{e^{\prime} \in I \geq(e)} X_{e^{\prime}, \tau} \leq C_{R T S / C T S},
$$

where constant $C_{R T S / C T S}=2 C_{1}$, and $I^{\geq}(e)$ is the links interfering with $e$ whose radius is not smaller than $e$.
Notice that above theorems hold for any multi-hop wireless networks in which both the transmission range and interference range could be heterogeneous and some links could be missing due to various reasons. If the interference range is homogeneous, then the constant $C_{\alpha}$ could be improved.

Let $\delta\left(F_{G}^{D 2}\right)$ be the maximum $\delta$-degree of all links $\mathbf{L}_{k}$ in the Step 2-3 of Algorithm 1. We now prove that Algorithm 1 has the following performance guarantee.

Theorem 7: Under RTS/CTS model, Algorithm 1 needs at most $2 C_{1} \cdot \delta_{\text {opt }}$ time-slots for all links without interference, where $\delta_{\text {opt }}$ is the minimum schedule period $T$.

Proof: Let $H$ be the vertex induced subgraph of $F_{G}^{D 2}$ such that each vertex in $H$ has degree at least $\delta\left(F_{G}^{D 2}\right)$. The existence of $H$ is straightforward from the definition of $\delta(G)$. Without loss of generality, let $\mathbf{L}_{i, j}$ be the vertex in $H$ with the smallest interference range. From Theorem 5, there exists a clique of size at least $\frac{\delta\left(F_{G}^{D 2}\right)+1}{2 C_{1}}$ in $F_{G}^{D 2}$. The optimal solution thus needs $\geq \frac{\delta\left(F_{G}^{D 2}\right)+1}{2 C_{1}}$ colors. Algorithm 1 uses $\leq \delta\left(F_{G}^{D 2}\right)+1$ colors. This finishes our proof.

\section{B. Scheduling under fPrIM Model}

Kumar et al. [7] studied the scheduling under a different protocol interference model (with parameter $\delta$ ): where a transmission by a node $v_{i}$ is successfully received by a node $v_{j}$ iff $\left\|v_{k}-v_{j}\right\| \geq(1+\delta)\left\|v_{i}-v_{j}\right\|$ for any node $v_{k} \neq v_{i}$. This needs every node to dynamically change its transmission power based on receiving node. Recall that in this paper, we assume that any node will have a fixed transmission power. It is not difficult to design network examples where the methods (processing links in the order of decreasing length) developed in [7] will not work under our model.

Under RTS/CTS model, we essentially showed that the optimal color assignment needs at least $\delta\left(F_{G}^{D 2}\right)$ colors. Note that when the graph is modeled by UDG, $\delta\left(F_{G}^{D 2}\right)$ is essentially $\Delta\left(F_{G}^{D 2}\right)$, where $\Delta\left(F_{G}^{D 2}\right)$ is the maximum degree of the conflict graph $F_{G}^{D 2}$. Thus, almost any greedy based coloring method (using at most $\Delta\left(F_{G}^{D 2}\right)+1$ colors) has a constant approximation ratio. Several previous literatures claimed the same result (that the optimal coloring needs $\Theta\left(\Delta\left(F_{G}^{P}\right)\right)$ colors) under the fPrIM model and proposed some algorithms to color the communication graph $G$ using $O\left(\Delta\left(F_{G}^{P}\right)\right)$ colors, where $\Delta\left(F_{G}^{P}\right)$ is the maximum degree of the conflict graph $F_{G}^{P}$ under fPrIM model. We can also define $\delta\left(F_{G}^{P}\right)$ as the maximum $\delta$-degree of the $F_{G}^{P}$ which can be computed by applying Step 2-3 of Algorithm 1 on $F_{G}^{P}$. However, as we will show later, there are examples of communication graphs whose optimal coloring needs constant colors, while, on the other hand, both $\Delta\left(F_{G}^{P}\right)$ and $\delta\left(F_{G}^{P}\right)$ are $O\left(n^{1-\epsilon}\right)$ for any $0 \leq \epsilon<1$ if all nodes have the same transmission range and $t_{i}=r_{i}=r$. This shows that any greedy algorithm that uses $\Theta\left(\Delta\left(F_{G}^{P}\right)\right)$ or even $\Theta\left(\delta\left(F_{G}^{P}\right)\right)$ colors could be very bad compared to the optimal solution.

We now describe such an example as in Figure 4. Here all nodes have same transmission range and interference range $r$. The links formed several groups such that all links in each group are parallel and each link has length $r$. The groups are placed in a cyclic manner such that any sender of one group interferes with all receivers in the previous group and does not interfere with any other receivers in other groups. The number of links in each group is $n^{1-\epsilon}$ and there are $n^{\epsilon}$ groups. Obviously, in the conflict graph $F_{G}^{P}$, the degree of each vertex (corresponding to 
a physical link) is $n^{1-\epsilon}$. Thus, $\Delta\left(F_{G}^{P}\right)=\delta\left(F_{G}^{P}\right)=n^{1-\epsilon}$. On the other hand, we can use at most 3 colors to color all the links without conflict: we color groups in clockwise order, and all links in the same group are assigned the same color that is the smallest available.

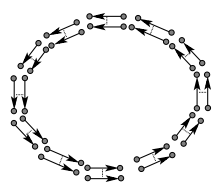

Fig. 4. Bad example for simple greedy

The above example shows that it is unclear whether Algorithm 1 can find a scheduling that approximates the optimal solution when the interference range equals the transmission range (the proof of Theorem 7 does not extend to this scenario). Fortunately, the ratio of the interference range over the transmission range is usually around 2 in practice. Next, we utilize this property to design an efficient link scheduling with a constant approximation ratio.

Given any two nodes $\mathbf{L}_{i, j}$ and $\mathbf{L}_{p, q}$ in conflict graph $F_{G}^{P}$ such that $v_{j}$ and $v_{q}$ are receivers, if $\mathbf{L}_{i, j}$ and $\mathbf{L}_{p, q}$ interfere with each other, then it is possible that (1) $v_{i}$ interferes $v_{q}$, or (2) $v_{p}$ interferes $v_{j}$, (3) or both. If $v_{p}$ interferes $v_{j}$, then we treat the link between $\mathbf{L}_{i, j}$ and $\mathbf{L}_{p, q}$ as an incoming link for $\mathbf{L}_{i, j}$. Similarly, if $v_{i}$ interferes $v_{q}$, we treat the link as an outgoing link for $\mathbf{L}_{i, j}$. Let $d_{i, j}^{i n}\left(F_{G}^{P}\right)$ and $d_{i, j}^{\text {out }}\left(F_{G}^{P}\right)$ be the incoming and outgoing degree of $\mathbf{L}_{i, j}$ in the conflict graph $F_{G}^{P}$ respectively. The number of incoming links of a vertex in $F_{G}^{P}$ is its incoming degree, and the number of outgoing links are its outgoing degree. Similarly, we define $\Delta^{\text {in }}\left(F_{G}^{P}\right)$ and $\Delta^{\text {out }}\left(F_{G}^{P}\right)$ as the maximum incoming and outgoing degree in graph $F_{G}^{P}$ respectively. When $\gamma_{i}>1$ for each node $v_{i}$, we can show that the optimal coloring needs at least $\Theta\left(\Delta^{i n}\left(F_{G}^{P}\right)\right)$ colors, where the hidden constant depending on $\min _{i} \gamma_{i}$ (which is typically 2 in practice).

Lemma 8: Consider any link $\mathbf{L}_{i, j}$, where $v_{j}$ is the receiver. Consider two links $\mathbf{L}_{p, q}$ and $\mathbf{L}_{s, t}$ that are $\mathbf{L}_{i, j}$ 's incoming links in conflict graph $F_{G}^{P}$, where $v_{q}$ and $v_{t}$ are the receivers. If $\angle v_{q} v_{j} v_{t} \leq \arcsin \frac{\gamma-1}{2 \gamma}$, then link $\mathbf{L}_{p, q}$ interferes with $\operatorname{link} \mathbf{L}_{s, t}$.

Proof: Due to space limit, the detailed proof is omitted. Please refer the Lemma 5 in the conference version [14].

Similar to Lemma 6, we have the following necessary condition for interference-free link scheduling under fPrIM model.

Lemma 9: For any time slot $\tau$, any valid interference-free link scheduling $\mathcal{S}$ under protocol interference model must satisfy that

$$
X_{e, \tau}+\sum_{e^{\prime} \in I^{i n}(e)} X_{e^{\prime}, \tau} \leq\left\lceil\frac{2 \pi}{\arcsin \frac{\gamma-1}{2 \gamma}}\right\rceil,
$$

where $I^{i n}(e)$ is the set of incoming links of $e$ that interfere $e$. This is because that for all incoming neighboring links of link $e$,

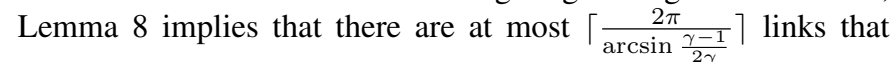
can be scheduled at any same time slot. Notice that when $\gamma=1$, $X_{e, \tau}+\sum_{e^{\prime} \in I(e)} X_{e^{\prime}, \tau}$ could be arbitrarily large as shown by a network example illustrated in Figure 4. In practice, $\gamma \geq 2$, which implies that $\left\lceil\frac{2 \pi}{\arcsin \frac{\gamma-1}{2 \gamma}}\right\rceil \leq 25$. We then present our main theorem about the optimum coloring for fPrIM model with $\gamma_{i}>1$.

Theorem 10: Optimal vertex coloring for conflict graph $F_{G}^{P}$ needs $\Theta\left(\Delta^{i n}\left(F_{G}^{P}\right)\right)$ colors if $\min _{i} \gamma_{i}$ is some constant $>1$.

Proof: For any link $\mathbf{L}_{i, j}$ such that $v_{j}$ is the receiver, we partition the space using $b$ equal-sized cones apexed at node $v_{j}$, where $b=\left\lceil\frac{2 \pi}{\arcsin \frac{\gamma-1}{2 \gamma}}\right\rceil$. From the Pigeon hole principle, $\mathbf{L}_{i, j}$ has at least $d_{i, j}^{i n}\left(F_{G}^{P}\right) / b$ links whose receivers are in the same cone. From Lemma 8, all links in the same cone interfere with each other. Thus, $\mathbf{L}_{i, j}$ has at least $d_{i, j}^{i n}\left(F_{G}^{P}\right) / b$ in-coming links such that they interfere with each other. It implies that any valid coloring will use at least $d_{i, j}^{i n}\left(F_{G}^{P}\right) / b$ among the incoming neighbors of link $\mathbf{L}_{i, j}$. Thus, the optimal coloring needs at least $\Delta^{i n}\left(F_{G}^{P}\right) / b+1$ colors.

Note that $\Delta\left(F_{G}^{P}\right)$ could be arbitrary larger than $\Delta^{i n}\left(F_{G}^{P}\right)$. Thus, simple greedy algorithm using $\Delta\left(F_{G}^{P}\right)$ colors does not work, e.g., the algorithm proposed in [1] for UDG networking model. It is known that the optimal coloring can be obtained by using greedy approach on a certain ordering of vertices in $F_{G}^{P}$. Next, with a careful selection of link ordering, we present our centralized scheduling method (Algorithm 2) that needs at most $2 \cdot \Delta^{i n}\left(F_{G}^{P}\right)+1$ colors which is asymptotically optimal.

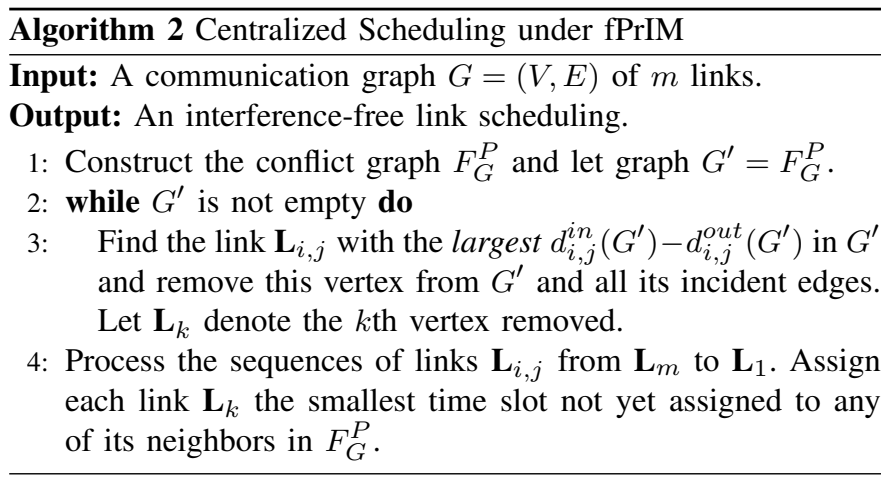

Theorem 11: Algorithm 2 uses at most $2 \cdot \Delta^{i n}\left(F_{G}^{P}\right)+1$ colors.

Proof: The key observation is that in any directed graph, the sum of all vertices' incoming degree equals the sum of outgoing degree. For the link $\mathbf{L}_{i, j}$ with the largest $d_{i, j}^{i n}\left(G^{\prime}\right)-d_{i, j}^{\text {out }}\left(G^{\prime}\right)$ in $G^{\prime}$, we must have $d_{i, j}^{i n}\left(G^{\prime}\right) \geq d_{i, j}^{\text {out }}\left(G^{\prime}\right)$. Thus, when we assign color (or time-slot) for the link $\mathbf{L}_{i, j}$, the subgraph induced by all the links that have already been processed is exactly the subgraph $G^{\prime}$ right before vertex $\mathbf{L}_{i, j}$ was removed in the while loop of Algorithm 2. Therefore, there are at most $2 \cdot d_{i, j}^{i n}\left(G^{\prime}\right)$ adjacent neighbors of $\mathbf{L}_{i, j}$ in $F_{G}^{P}$ that have already been processed. In other words, the smallest time-slot assigned to $\mathbf{L}_{i, j}$ is at most $2 \cdot d_{i, j}^{i n}\left(G^{\prime}\right)+1$, which is at most $2 \cdot d_{i, j}^{i n}\left(F_{G}^{P}\right)+1$. This proves that we need at most $2 \cdot \Delta^{i n}\left(F_{G}^{P}\right)+1$ time-slots for an interferencefree schedule.

\section{Distributed Link SCHEduling}

In a wireless network, centralized algorithm may not be possible and even if possible, due to the dynamic features of wireless networks, it is inefficient to update the coloring using a centralized algorithm. Thus, in this section, we design efficient distributed algorithms to get a valid coloring with good performance guarantee.

\section{A. Scheduling under RTS/CTS Model}

In literatures, several distributed algorithms have been proposed for the vertex coloring. The first solution is to simply apply a distributed vertex coloring on the conflict graph $F_{G}^{D 2}$. For arbitrary graphs, a $\Delta+1$-coloring can be computed in time $O\left(\log ^{*} n+\Delta^{2}\right)$ or $O(\Delta \log n)$ [8], [20]. Recall that all previous distributed algorithms work for the general graph. By taking advantage of special properties of conflict graph defined here, we 
are able to obtain a deterministic distributed coloring algorithm that colors the links with $O\left(\Delta\left(F_{G}^{D}\right)\right)$ colors in almost constant time when the interference ranges are homogeneous. On the other hand, as shown in our centralized algorithm, the optimal color is $\Theta\left(\delta\left(F_{G}^{D 2}\right)\right)$ which could be much smaller than $\Delta\left(F_{G}^{D 2}\right)$ when interference ranges are heterogeneous. Thus, simply applying a coloring algorithm with ratio $\Theta\left(\Delta\left(F_{G}^{D 2}\right)\right)$ may not achieve a good performance. The first instinct is to design a distributed version of Algorithm 1. However, finding the node with the global maximum degree iteratively does not seem promising for distributed algorithm. Thus, we need to find some lower bound for the optimal color other than $O\left(\delta\left(F_{G}^{D 2}\right)\right)$.

Given two nodes $v_{i}$ and $v_{j}$, we say that $v_{i}$ precedes $v_{j}$ if and only if $r_{i}>r_{j}$ or $r_{i}=r_{j}$ and $i>j$. Given a pair of links $\mathbf{L}_{i, j}$ and $\mathbf{L}_{p, q}$ with different heads $h_{i, j} \neq h_{p, q}$, we say that $\mathbf{L}_{i, j}$ precedes $\mathbf{L}_{p, q}$ if $r_{i, j}>r_{p, q}$ or $r_{i, j}=r_{p, q}$ and $h_{i, j}>h_{p, q}$. Recall that $r_{i, j}=\max \left\{r_{i}, r_{j}\right\}$. We also say that the corresponding vertex $\mathbf{L}_{i, j}$ precedes $\mathbf{L}_{p, q}$ in the conflict graph in this case. For a vertex $\mathbf{L}_{i, j}$ in graph $F_{G}^{D 2}$, let $d_{i, j}^{\geq}\left(F_{G}^{D 2}\right)$ be the number of adjacent vertices that precede $\mathbf{L}_{i, j}$, which is called efficient degree of $\mathbf{L}_{i, j}$. From Theorem 5, there are at least $d_{i, j}^{\geq}\left(F_{G}^{D 2}\right) /\left(2 C_{1}\right)$ vertices adjacent to and preceding $\mathbf{L}_{i, j}$ that form a clique in which each vertex (i.e., the corresponding link in the communication graph) interferes with each other. Let $\phi\left(F_{G}^{D 2}\right)=\max _{\mathbf{L}_{i, j}} d_{i, j}^{\geq}\left(F_{G}^{D 2}\right)$, then Theorem 5 shows that optimal coloring algorithm needs at least $\phi\left(F_{G}^{D 2}\right) /\left(2 C_{1}\right)$ colors. Thus, finding a coloring algorithm using at most $\Theta\left(\phi\left(F_{G}^{D 2}\right)\right)$ colors is a constant-ratio approximation algorithm. Unlike the centralized Algorithm 1 in which the lower bound of $\delta\left(F_{G}^{D 2}\right)$ could not be found by using only local information, the lower bound of $\phi\left(F_{G}^{D}\right)$ could be easily obtained by any link $\mathbf{L}_{i, j}$ by simply counting the number of interfering links that precede itself, i.e., with larger link interference radius. Algorithm 3 presents our distributed coloring method that uses at most $\phi\left(F_{G}^{D 2}\right)$ colors.

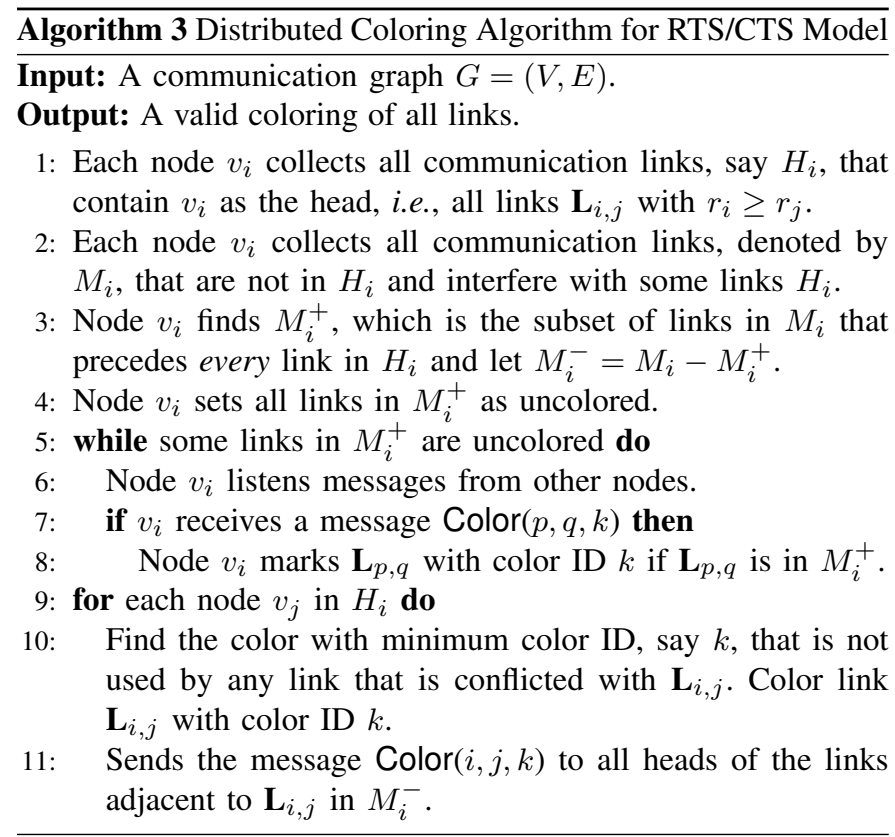

Theorem 12: Algorithm 3 computes a valid coloring using at most $\phi\left(F_{G}^{D 2}\right)$ colors, which is asymptotically optimal.

Proof: First, we show that the algorithm does terminate.
Since it is straightforward that the number of nodes in $H_{i}$ is bounded by $\phi\left(F_{G}^{D}\right)$, the for loop terminates in $O(n)$ iterations. Thus, the maximum time needed for all other processes other than while loop is bounded by a finite time $T$ and our main focus is to show that the while loop does terminate for any node $v_{i}$. Let $\left(v_{\sigma_{1}}, v_{\sigma_{2}}, \ldots, v_{\sigma_{n}}\right)$ be the sorted list of nodes in the decreasing order of their interference range. Thus, $v_{\sigma_{i}}$ precedes $v_{\sigma_{j}}$ if and only if $i<j$. Since $v_{\sigma_{1}}$ precedes every other nodes, $M_{\sigma_{1}}^{+}$is empty and $v_{\sigma_{1}}$ colors all links that are adjacent to $v_{\sigma_{1}}$ in time $T$. Now consider the node $v_{\sigma_{2}}$ and $M_{\sigma_{2}}^{+}$. If $\mathbf{L}_{p, q} \in M_{\sigma_{2}}^{+}$, then either $v_{p}$ or $v_{q}$ is $v_{\sigma_{1}}$. Thus, all links in $M_{\sigma_{2}}^{+}$are colored. Therefore, all links that are adjacent to $v_{\sigma_{2}}$ are colored before time $2 T$. Similarly, all links that are adjacent to $v_{\sigma_{j}}$ are colored before time $j \cdot T$. Thus, all links are colored in time $n \cdot T$. It is straightforward to show that, by assuming color one link takes a unit time, the running time of this algorithm is at most $m$, where $m$ is the number of directed communication links.

Second, we show that the computed coloring is valid, i.e., no two conflict links have the same color. Consider conflict links $\mathbf{L}_{i, j}$ and $\mathbf{L}_{p, q}$, following we discuss by cases.

Case 1: $\mathbf{L}_{i, j}$ and $\mathbf{L}_{p, q}$ have the same head. Without loss of generality, we assume that $v_{i}=v_{p}$ is the head of the links. Thus, both $\mathbf{L}_{i, j}$ and $\mathbf{L}_{p, q}$ are in $H_{i}$. Therefore, $\mathbf{L}_{i, j}$ and $\mathbf{L}_{p, q}$ have different colors.

Case 2: $\mathbf{L}_{i, j}$ and $\mathbf{L}_{p, q}$ have different heads. Then, without loss of generality, we can assume that $h_{i, j}=i, h_{p, q}=p$ and $v_{i}$ precedes $v_{p}$. Since $\mathbf{L}_{i, j} \in M_{p}^{+}, \mathbf{L}_{i, j}$ is colored before $M_{p}^{+}$ becomes empty. Thus, $\mathbf{L}_{p, q}$ is colored after $\mathbf{L}_{i, j}$ is. Therefore, when $v_{p}$ colors $\mathbf{L}_{p, q}$, it uses a color that is different from the color of $\mathbf{L}_{i, j}$ based on our algorithm.

Third, it is straightforward that Algorithm 3 uses at most $\phi\left(F_{G}^{D 2}\right)$ colors, i.e., it has a constant approximation ratio.

Notice that in Algorithm 3, we start to color a link after all interfering links preceding it are colored. Thus, in the worst case, it may take time $O(n)$ to color all the links, where $n$ is the number of nodes in the network. Here we assume that in one time unit, a node can color all its incident links. Comparing with previous poly-logarithmic time distributed coloring algorithms that color the graph using $\Delta\left(F_{G}^{D 2}\right)$ colors, Algorithm 3 may take longer time. However, following example shows that $\Delta\left(F_{G}^{D}\right)$ could be

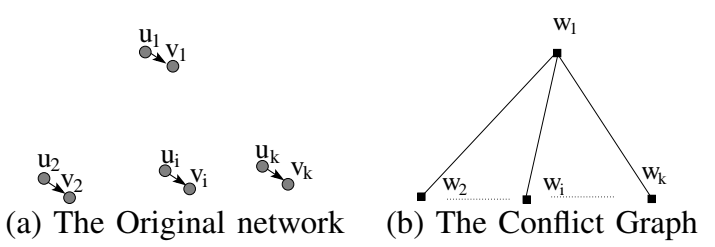

Fig. 5. $\Delta$ could be $\Theta(n)$ of number of colors used by Alg. 3 .

as large as $O(n)$ times of the color used by Algorithm 3, where $n$ is the number of the nodes in original network. In Figure 5(a), there are $k$ pairs of transmission links $u_{1} v_{1}, \ldots, u_{n} v_{n}$. Nodes $u_{1}, v_{1}$ have interference range 1 and all other nodes have interference range $\epsilon$, where $\epsilon$ is a small positive constant such that node $u_{i}$ does not interfere $v_{j}$ for $i, j>1$. The corresponding conflict graph is shown in Figure 5(b). It is not difficult to see that we only need two colors while the degree of $\mathbf{L}_{1,1}$ is $n-1$. In other words, compared with previous poly-logarithmic time methods with $\Omega(n)$ approximation ratios, our method has a constant approximation ratio using larger worst-case running time. 


\section{B. Faster Scheduling under RTS/CTS Model}

Although Algorithm 3 computes a coloring that is at most constant times of the optimal, it may need linear number of rounds to compute the coloring. In certain circumstances, we would prefer the distributed algorithms that run fast to the distributed algorithms that have good performance as long as the fast distributed algorithm does not perform much worse. Following we present another distributed algorithm that computes the coloring very fast with a good performance guarantee of $O(\log (\psi)+1)$, where $\psi$ is the ratio between the maximum interference range over the minimum interference range among all nodes.

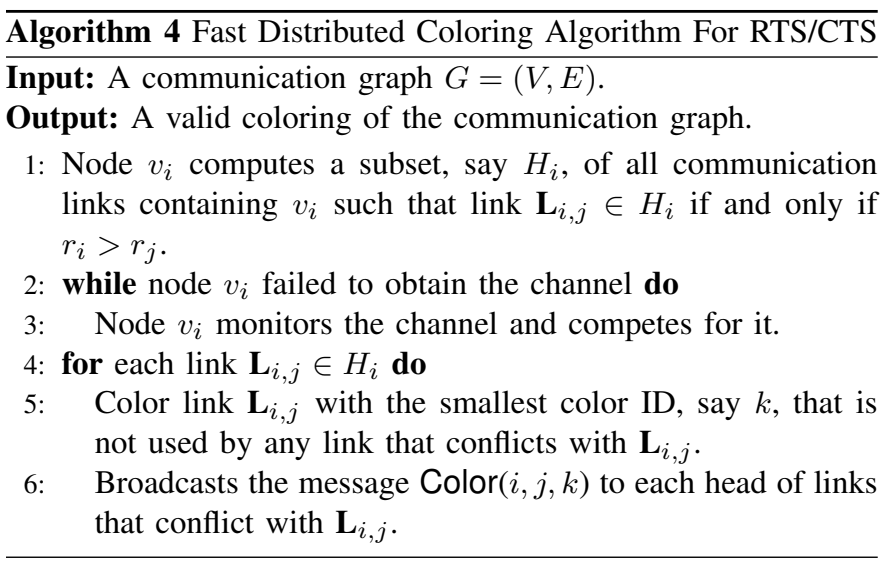

Algorithm 4 assumes that there is certain competition based MAC layer (e.g., 802.11 with RTS/CTS) available for a node to obtain the channel. We use this MAC mechanism to obtain a link scheduling that is efficient and interference free. Algorithm 4 is very simple and can be implemented without much additional computation on each node. However, the proof of the performance guarantee is not straightforward. To prove the main theorem, we need some notation in order to extend the Theorem 4 and Theorem 5. For a given node $v_{k}$, Let $N^{\geq}\left(v_{k}, \alpha, \beta\right)$ be a node set composed of the nodes satisfying that (1) each of their interference radius is at least $\frac{r_{k}}{\beta}$; (2) each of them interferes some nodes in $R\left(v_{k}, \alpha\right)$. Let $N^{\geq}\left(\mathbf{L}_{i, j}, \alpha, \beta\right)$ be the union of $N^{\geq}\left(v_{i}, \alpha, \beta\right)$ and $N^{\geq}\left(v_{j}, \alpha, \beta\right)$. The proofs of the following Lemma 13 and 14 are similar to the proofs of Theorem 4 and 5 respectively and thus are omitted here.

Lemma 13: For any node $v_{k}$ and any set $V_{k} \subseteq N^{\geq}\left(v_{k}, \alpha, \beta\right)$, there exists a subset $V_{k}^{\prime}$ of $V_{k}$ with cardinality at least $\left\lceil\left|V_{k}\right| / C_{\alpha, \beta}\right\rceil$ such that nodes in $V_{k}^{\prime}$ interfere with each other where $C_{\alpha, \beta}=$ $(6 \alpha \beta+1)^{2}+11$.

Lemma 14: For any link $\mathbf{L}_{i, j}$ and any set $V_{i j} \subseteq$ $N^{\geq}\left(\mathbf{L}_{i, j}, \alpha, \beta\right)$, there exists a subset $V_{i j}^{\prime}$ of $V_{i j}$ with cardinality at least $\left\lceil V_{i j} /\left(2 C_{\alpha+1, \beta}\right)\right\rceil$ such that links in $V_{i j}^{\prime}$ interfere with each other.

Let $\Delta(\alpha, \beta)=\max _{\mathbf{L}_{i, j}}\left|N^{\geq}\left(\mathbf{L}_{i, j}, \alpha, \beta\right)\right|$ and $\chi\left(F_{G}^{D 2}\right)$ be the optimal number of colors. Based on Lemma 14, the following theorem is straightforward, for any fixed $\alpha, \beta$,

Theorem 15: $\chi\left(F_{G}^{D}\right) \geq\left\lceil\Delta(\alpha, \beta) /\left(2 C_{\alpha+1, \beta}\right)\right\rceil$.

We then present our main theorem for our fast distributed coloring method.

Theorem 16: Algorithm 4 computes a coloring that is at most $O(\log (\psi)+1)$ times of optimum $\chi\left(F_{G}^{D 2}\right)$.

Proof: Without loss of generality, let link $\mathbf{L}_{i, j}$ be the link that has the maximum color ID, say g. To prove the theorem, we will show that $\mathbf{g} \leq 2 C_{1,2} \cdot(\log (\psi)+1) \cdot \chi$. Following we prove it by contradiction and for the sake of contradiction, assume that g $>2 C_{1,2} \cdot(\log (\psi)+1) \cdot \chi$.

We first argue that for any $0 \leq k \leq \log (\psi)$, there exists a link $\mathbf{L}_{i^{(k)}, j^{(k)}}$ such that $r_{i^{(k)}, j^{(k)}}<r_{i, j} / 2^{k}$ and its color ID is not smaller than $\mathbf{g}-2 C_{1,2} \cdot k \cdot \chi$. We prove this argument by induction on $k$. If $k=0$, then the argument trivially holds. Assume for $k \leq p$, the argument holds. From Theorem 15, by letting $\alpha=0$ and $\beta=2, \chi \geq \Delta(0,2) /\left(2 C_{1,2}\right)$. In other words, the number of links, that interfere or are interfered by link $\mathbf{L}_{i(p), j(p)}$ and whose radius is not smaller than $r_{i(p), j(p)} / 2$, is at most $2 C_{1,2} \cdot \chi$. Thus, there must exist a link $\mathbf{L}_{i(p+1), j(p+1)}$ such that

1) $\mathbf{L}_{i^{(p+1)}, j^{(p+1)}}$ interferes or is interfered by $\mathbf{L}_{i^{(p)}, j^{(p)}}$;

2) $r_{i(p+1), j(p+1)}<r_{i, j} / 2^{p+1}$; and

3) $\mathbf{L}_{i(p+1), j(p+1)}$ 's color ID is at least $\mathbf{g}-2 C_{1,2} \cdot(p+1) \cdot \chi$.

This finishes the induction.

Thus, let $k=\lfloor\log (\psi)\rfloor$, link $\mathbf{L}_{i\lfloor\log (\psi)\rfloor, j}\lfloor\log (\psi)\rfloor$ has the color ID not smaller than $\mathbf{g}-2 C_{1,2} \cdot\lfloor\log (\psi)\rfloor \cdot \chi$. This implies that $\mathbf{L}_{i\lfloor\log (\psi)\rfloor, j\lfloor\log (\psi)\rfloor}$ has at least $2 C_{1,2} \cdot \chi+1$ adjacent links. Since, $r_{i(\lfloor\log (\psi)\rfloor), j(\lfloor\log (\psi)\rfloor)}<r_{i, j} / 2^{\lfloor\log (\psi)\rfloor)}$ and $r_{p, q} \geq r_{i, j} / 2^{\log (\psi)}$, all links that interfere or are interfered by $\operatorname{link} \mathbf{L}_{i}\lfloor\log (\psi)\rfloor, j\lfloor\log (\psi)\rfloor$ have interference radius at least $r_{i\lfloor\log (\psi)\rfloor, j}\lfloor\log (\psi)\rfloor / 2$. From Lemma 14, $\chi \geq\left\lceil\frac{2 C_{1,2} \cdot \chi+1}{2 C_{1,2}}\right\rceil \geq \chi+1$, which is a contradiction. Thus, $\mathbf{g} \leq$ $2 C_{1,2} \cdot(\log (\psi)+1) \cdot \chi$. This finishes the proof.

Algorithm 4 essentially is a First-Fit coloring method. It has been proved in [21] that, any First-Fit coloring of an $d$-inductive graph with $n$ nodes will produce a coloring using colors at most $O(d \log n)$ times of the optimum. Here a graph $G$ is $d$-inductive if we can number the vertices such that each node has at most $d$ edges connected to the nodes with larger numbers. We essentially proved previously that graphs $F_{G}^{P}$ and $F_{G}^{D 2}$ are $d$-inductive graphs for some constants $d$. Thus, we have the following theorem.

Theorem 17: Algorithm 4 computes a coloring that is at most $O\left(\min (\log n, 1+\log \psi)\right.$ times of optimum $\chi\left(F_{G}^{D 2}\right)$.

Notice that, in Algorithm 4, a node can start assigning timeslots to its incident links as long as it obtained the communication channel. Thus, the time complexity of this algorithm will be much close to the node coloring number of the communication graph $G$, in which two interfering nodes should be assigned different colors. Notice that, it was proved in [22] that for disk graphs, the tight bound for approximation ratio for online coloring of disk graphs is $\min \{\log n, \log \psi\}$. Thus, we know that it is impossible to design distributed algorithm for link scheduling with better asymptotic approximation ratio when no any ordering are allowed among links.

\section{Scheduling under fPrIM Model}

From Theorem 11, any coloring algorithm that uses $O\left(\Delta^{i n}\left(F_{G}^{P}\right)\right)$ colors under the fPrIM model has a constant approximation ratio. Here we give a distributed algorithm (Algorithm 5) that bears the similar idea of our centralized method (Algorithm 2).

Theorem 18: Algorithm 5 computes a valid coloring with at most $2 \cdot \Delta^{i n}\left(F_{G}^{P}\right)+1$ colors with $O(m)$ messages, where $m$ is the number of communication links.

Proof: Notice that for each link $\mathbf{L}_{i, j}$, Algorithm 5 uses the smallest color that is not used by any links in $S_{i, j}$. Since the number of incoming links is not smaller than the outgoing links in $S_{i, j}$, link $\mathbf{L}_{i, j}$ is colored with a color not greater than 


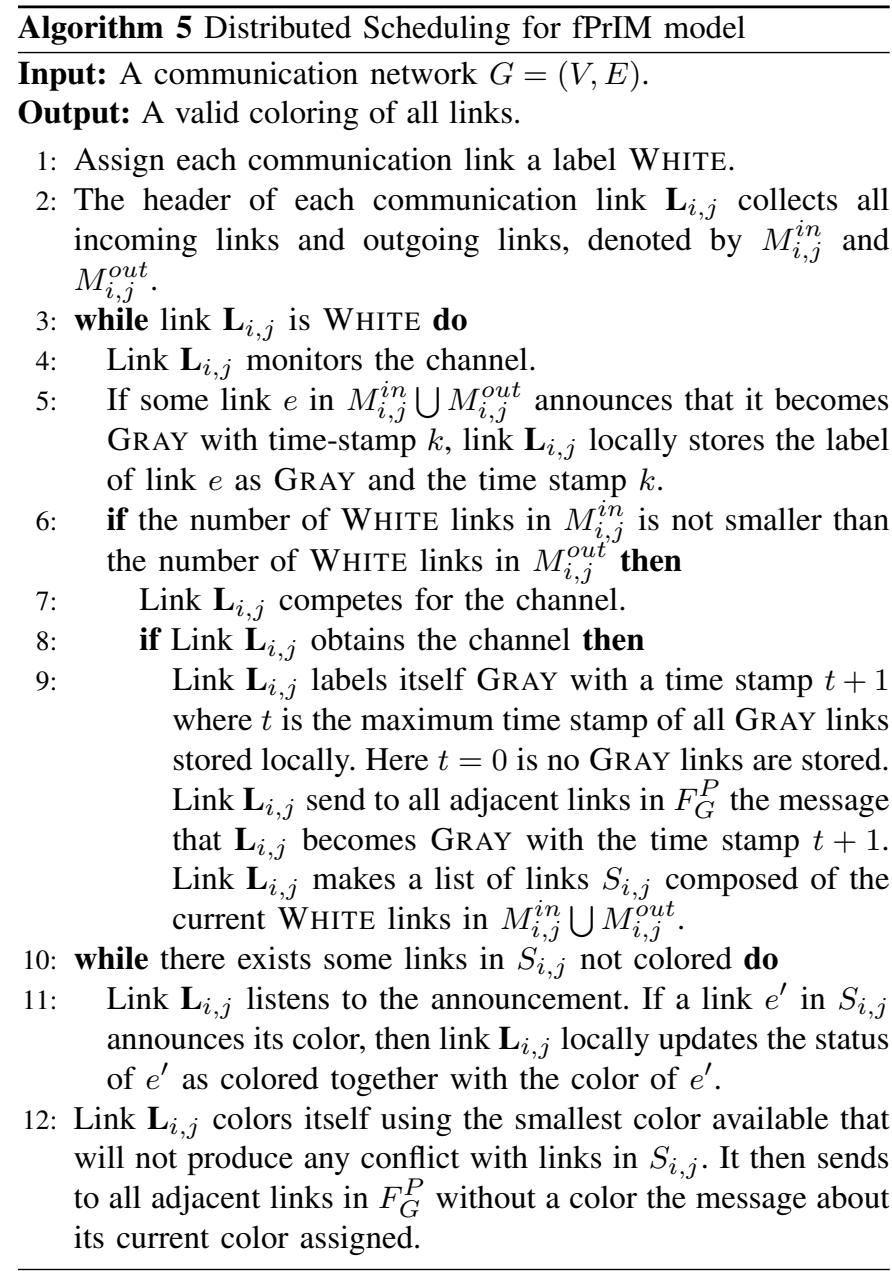

$2 \cdot d_{i, j}^{i n}\left(F_{G}^{P}\right)+1$. Thus, Algorithm 5 computes a valid coloring with at most $2 \cdot \Delta^{i n}\left(F_{G}^{P}\right)+1$ colors. Note that each link $\mathbf{L}_{i, j}$ only announces twice in our distributed scheduling algorithm: when it becomes GRAY and when it is colored. Thus, the overall message complexity is $O(m)$.

Notice that our faster distributed algorithm for RTS/CTS interference model can also be used for the fixed power protocolinterference model here. Using similar proof techniques, we can also prove the following result: our faster distributed coloring algorithm computes a coloring that is at $\operatorname{most} O(\min (\log n, 1+$ $\log \psi)$ times of optimum $\chi\left(F_{G}^{P}\right)$.

\section{Scheduling with Traffic and Schedulable Flows}

\section{A. Scheduling With Traffic Load}

In TDMA system, the minimization of the number of colors is closely related to the maximization of the network throughput. One intrinsic assumption behind the idea of coloring is that each communication link has the same packet arrive rate, i.e., the number of traffics that need to go through each communication link is same. However, this is not likely to be true and it is possible that some communication link carries more traffic than others, e.g., when joint routing and link scheduling is performed. In [14], we show that simple adaptation of minimum coloring to schedule link transmissions will produce a network throughput that is arbitrarily smaller than the optimum. Thus, we need to generalize the coloring that can take the traffic rate on each communication link into account. In this paper, we use the weighted coloring to capture this, which is defined as follows.

Definition 1: Given a graph $G=(V, E)$ where $V$ is the set of vertices and $E$ is the set of links. Every link $e_{i} \in E$ has an integral weight $w_{i} \geq 0$. A weighted link coloring is an assignment of at least $w_{i}$ distinct colors to each link $e_{i}$ such that no two links sharing the same color interfere with each other.

By introducing the notation of weighted coloring, we can assign different weight to different communication links. For example, given a set of $k$ flow requirements $f_{i}$ from $s_{i}$ to $t_{i}, 1 \leq i \leq k$, a certain routing algorithm will determine the routing path for each flow. The weight of a link $e$ is then the total flow passing through $e$ divided by the bandwidth $\mathbf{c}(e)$ of link $e$. Following, we show how to obtain a valid weighted coloring based on the unweighted coloring (Algorithm 6).

$\overline{\text { Algorithm } 6 \text { Weighted Coloring Algorithm Based on Unweighted }}$
Coloring Algorithm $\mathcal{A}$
Coloring Algorithm $\mathcal{A}$

Input: A communication graph $G=(V, E)$ with weight on each link and an unweighted coloring algorithm $\mathcal{A}$.

Output: A valid coloring of the links.

1: Build the conflict graph $F_{G}$ based on original graph $G$ and interference model. Assign weight $w_{i, j}$ to vertex $\mathbf{L}_{i, j} \in F_{G}$.

2: Construct a new conflict graph $F_{G}^{\prime}$ from $F_{G}$ as follows: for each vertex $\mathbf{L}_{i, j}$ with weight $w_{i, j}$, we create $w_{i, j}$ vertices, $\mathbf{L}_{i, j}^{1}, \mathbf{L}_{i, j}^{2}, \ldots, \mathbf{L}_{i, j}^{w_{i, j}}$ and add them to $F_{G}^{\prime}$. Add to graph $F_{G}^{\prime}$ the edges connecting $\mathbf{L}_{i, j}^{a}, \mathbf{L}_{i, j}^{b}$ for $1 \leq a<b \leq w_{i, j}$. Add to graph $F_{G}^{\prime}$ an edge between $\mathbf{L}_{i, j}^{a}$ and $\mathbf{L}_{p, q}^{b}$ if and only if there is an edge between $\mathbf{L}_{i, j}$ and $\mathbf{L}_{p, q}$ in graph $F_{G}$.

3: Run the unweighted vertex coloring algorithm $\mathcal{A}$ on $F_{G}^{\prime}$.

4: Assign link $\mathbf{L}_{i, j}$ all the colors that are used by $\mathbf{L}_{i, j}^{k}$ for $1 \leq$ $k \leq w_{i, j}$ in $F_{G}^{\prime}$.

We show Algorithm 6 has a performance guarantee that is not worse than that of the unweighted coloring algorithm $\mathcal{A}$.

Theorem 19: If $\mathcal{A}$ uses at most $\alpha$ times of the optimal colors for unweighted coloring, then Algorithm 6 also needs at most $\alpha$ times of the optimal colors for weighted coloring.

Proof: Notice that for any valid weighted coloring for $F_{G}$, $\mathbf{L}_{i, j}$ is assigned at least $w_{i, j}$ colors. By assigning each vertex $\mathbf{L}_{i, j}^{k}$ in $F_{G}^{\prime}$ a distinct color that is assigned to $\mathbf{L}_{i, j}$, we obtain a valid unweighted coloring for $F_{G}^{\prime}$. Thus, $\chi\left(F_{G}^{\prime}\right) \leq \chi\left(F_{G}\right)$. Here $\chi\left(F_{G}^{\prime}\right)$ is the minimum number of colors needed for unweighted coloring in $F_{G}^{\prime}$ and $\chi\left(F_{G}\right)$ is the minimum number colors needed for weighted coloring in $F_{G}$. Since $\mathcal{A}$ will return a coloring with at most $\alpha \cdot \chi\left(F_{G}^{\prime}\right)$ colors, Algorithm 6 produces a coloring with at most $\alpha \cdot \chi\left(F_{G}^{\prime}\right) \leq \alpha \cdot \chi\left(F_{G}\right)$ colors. This finishes the proof.

The basic idea of Algorithm 6 is to create a clique of size $w_{i, j}$ for each link $\mathbf{L}_{i, j}$ and color the new graph using unweighted coloring method $\mathcal{A}$. Although this gives a general framework to design weighted coloring, its time-complexity could be large if the weight is large. Fortunately, Algorithm 6 could be simplified without much overhead compared to the unweighted algorithm: the main idea is to assign colors for one link at once: instead of assigning one time-slot to a link $\mathbf{L}_{k}$, we assign $w_{k}$ time-slots to link $\mathbf{L}_{k}$ when process link $\mathbf{L}_{k}$. As an example, we modify the Algorithm 4 to obtain a fast weighted coloring (Algorithm 7). Following we show that Algorithm 7 has the same performance guarantee as Algorithm 4. 


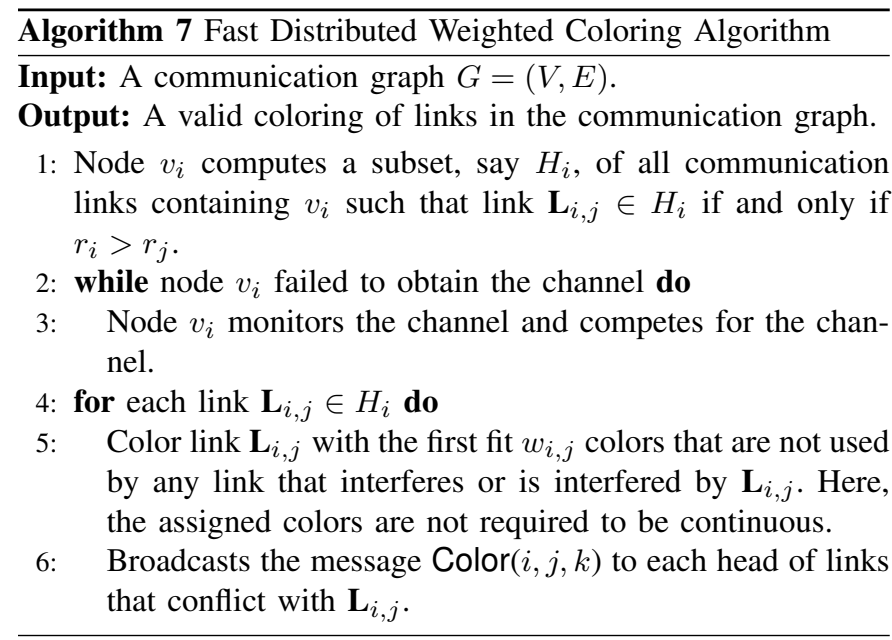

Theorem 20: Algorithm 7 finds a coloring that needs at most $O(\log (\psi)+1)$ times of optimum.

Proof: Let $\mathcal{A}_{w}$ be the coloring algorithm by applying Algorithm 6 based on Algorithm 4. Observe that the coloring of $\mathcal{A}_{w}$ is nondeterministic, i.e., the output could be different because of the randomization introduced by the different processing time of different nodes. However, it is true that the output of Algorithm 7 is one of the possible outputs of $\mathcal{A}_{w}$. From Theorem 19, any coloring output by $\mathcal{A}_{w}$ is at most $O(\log (\psi)+1)$ times the optimal. Thus, Algorithm 7 computes a coloring that needs at most $O(\log (\psi)+1)$ times optimal color.

Similarly, we can modify Algorithm 1 and Algorithm 3 to obtain efficient weighted coloring methods with the same time complexities and approximation ratios. Theorem 1 directly follows from the above two theorems.

\section{B. Necessary and Sufficient Conditions for Schedulable Flows}

Similar to [1], [3], [7], we also make the connection with flows on the links of a wireless network $G$ and the link scheduling. We give both a necessary and a sufficient condition on the link flows such that an interference-free link scheduling is feasible. Recall that we use $f(e), \mathbf{c}(e)$ to denote the load and the capacity of a link $e$ respectively. From Lemma 6 and Theorem 7, it follows that

Theorem 21: Under the RTS/CTS model, any link flow $f$ that permits an interference-free link scheduling must satisfy the constraint $\frac{f(e)}{\mathbf{c}(e)}+\sum_{e^{\prime} \in I \geq(e)} \frac{f\left(e^{\prime}\right)}{\mathbf{c}\left(e^{\prime}\right)} \leq 2 C_{1}$. On the other hand, if

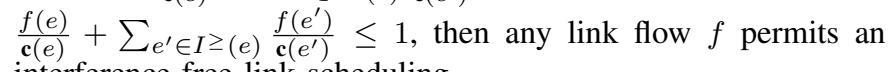
interference-free link scheduling.

Similarly, under the fPrIM Model, we have

Theorem 22: Under the fPrIM model, any link flow $f$ that permits an interference-free link scheduling must satisfy the

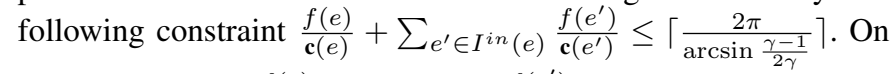
the other hand, if $\frac{f(e)}{\mathbf{c}(e)}+\sum_{e^{\prime} \in I^{i n}(e)} \frac{f\left(e^{\prime}\right)}{\mathbf{c}\left(e^{\prime}\right)} \leq 1$, then any link flow $f$ permits an interference-free link scheduling.

The proofs of the above theorems are similar to those of [1], [3], [7] for other interference and networking models, and are thus omitted here.

\section{ViI. Performance Evaluation of Scheduling}

Evaluation of OuR Scheduling Algorithms: We first evaluate the performances of our scheduling algorithms for RTS/CTS model via simulations with random networks.
Network Settings: In these simulations, we randomly generate $n$ wireless nodes uniformly in a $10 \times 10$ unit region. The transmission range is randomly drawn from 1.8 to 2 unit, while the interference range is randomly set to be 1.5 to 2 times of its transmission range. Typically, a unit represents about 50 meters here. We assume there is a sink (or an access point) in the network, all traffics are towards it. The sink is placed in the center of the region in the simulations. We vary the node number $n$ from 40 to 200 . For each number $n, 100$ vertex sets (networks) are randomly generated. Given a sampled network, we not only test the number of colors and the network throughput resulted by our various link scheduling algorithms, but also count the number of messages and rounds used by the distributed algorithms. The average of these performances over all these 100 randomly sampled networks are reported. For each source, we run the classical shortest path algorithm to determine the traffic route. Notice that our scheduling algorithms do not rely on any particular routing algorithms, here the shortest path routing is used as an example.

In the first scenario, we assume the system does not know the volume of each traffic. So it is an unweighted case where we need to assign one color for each link involved in the traffics. We test our centralized and two distributed algorithms (Algorithm 1 [Cent], Algorithm 3 [Dist-1], and Algorithm 4 [Dist2]). The simulation results are reported in the upper row of Figure 6. First, for the number of colors and the throughput, three algorithms have similar performances. When the node number increases, more colors are needed and the throughput decreases. The centralized algorithm has the best throughput while the fast distributed algorithm has the worst, as our expectation. For both distributed algorithms we also count the number of messages and rounds used. It shows that Dist-1 algorithm used much more messages and rounds than Dist-2 (fast distributed algorithm). The large number of rounds and messages needed by Dist- 1 is due to the first two steps in Algorithm 3, which collect all communication links in $H_{i}$ and $M_{i}$. The large number of rounds of Dist-1 is mainly due to conflicts among messages for collecting information. Notice that two adjacent links in the conflict graph need to compete for the channel first. After a node $v_{i}$ obtained the channel, it uses a unit of time to assign colors to all links in $H_{i}$ and inform other interfering links about the coloring used.

In the second scenario, we randomly draw the traffic produced by each node from 1 to 10 units. Then for each link $\mathbf{L}_{i, j}$, its weight $w_{i, j}$ is the total volumes of traffics that need to go through it, which could be 0 . The simulation results are given in the lower row of Figure 6. The throughput of weighted methods are much better than those of unweighted methods. Our centralized and distributed methods have similar throughput.

Benefits of Our Scheduling Methods: We then evaluate the performances of our distributed link scheduling algorithms by conducting simulations in QualNet 3.9 [23]. Notice that Algorithm 4 is a special case of Algorithm 7. Thus, we only evaluate the performance of Algorithm 7 (Fast Distributed Weighted Coloring Algorithm) based on RTS/CTS model, hereafter called FDWCA, by comparing it with DSR [24] and AODV [25] approach. In FDWCA, we run the classical shortest path algorithm to determine the routing path. Here, our goal is to show that proper link scheduling can conserve energies and improve network performance. DSR and AODV are contention-based, without link scheduling. 

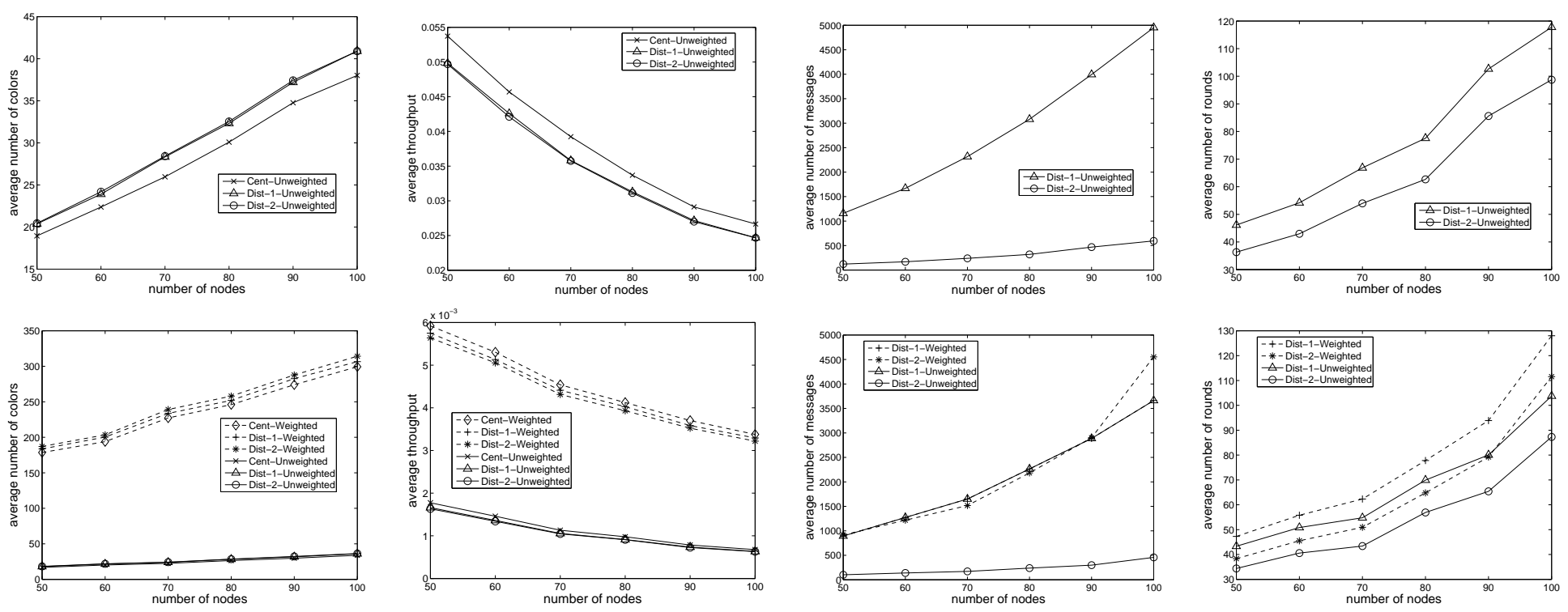

Fig. 6. Upper row: scheduling without traffic load information. Lower row: Scheduling with nonuniform traffic load.

Network Settings: We randomly generate $n$ nodes in $1000 \mathrm{~m} \times$ $1000 \mathrm{~m}$ square area, and adopt $802.11 b$ as physical and MAC layer model. In $802.11 b$ model, the transmission data rate is set to $2 \mathrm{Mbps}$, and maximum transmission power is $15.0 \mathrm{dBm}$ and receive sensitivity is $-89.0 \mathrm{dBm}$. We simulate periodical traffic from all nodes to a single sink using CBR (Constant Bit Rate) scenario, with packet size 128 bytes each. The slot duration is set to $10 \mathrm{~ms}$ in FDWCA. We evaluate different methods by comparing packet delay, average energy consumption, packet delivery ratio, and network throughput. Clearly, the delay and delivery ratio criteria reflect the network throughput. Higher packet delivery ratio and lower packet delay mean better network throughput. Average energy consumption is the average energy cost to delivery a certain number of packets from sources to sink. We calculate the average energy consumption of all nodes.

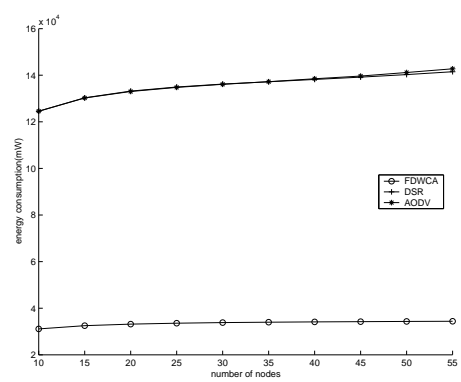

(a) Packet delay

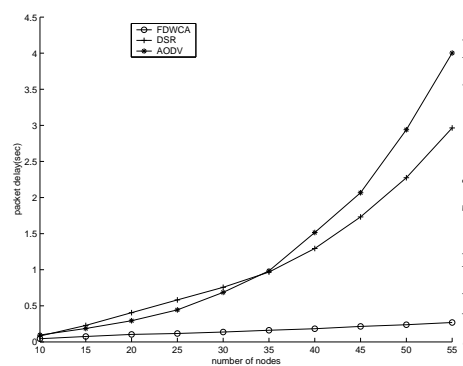

(b) Energy consumption
Fig. 7. Packet delay and energy consumption per round, when the network varies size in $[10,55]$ range and fixes the reporting interval at 5 seconds.

We first evaluate the packet delay and energy consumption, by fixing the reporting interval at 5 second and varying the network size from 10 to 55 . For each specific network size, we generate 50 samples to calculate the average performance. The results are shown in Figure 7. In Figure 7(a), as the network size grows linearly, packet delays in DSR and AODV approaches grow exponentially, while it increases near linearly in our FDWCA algorithm. In DSR and AODV, the large delay is mainly caused by the random resource competition of nodes, since every node is trying to send data to its parent node and eventually to the sink. The communication is not coordinated. Figure 7(b) shows the comparison of average energy consumption for these three approaches. The advantage of FDWCA is obvious. DSR and AODV cost more energy than FDWCA, because enormous media contention wastes energies.

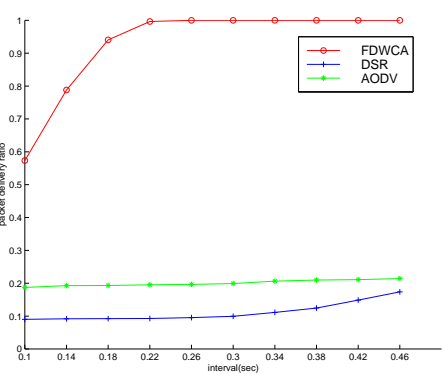

(a) Packet delivery ratio

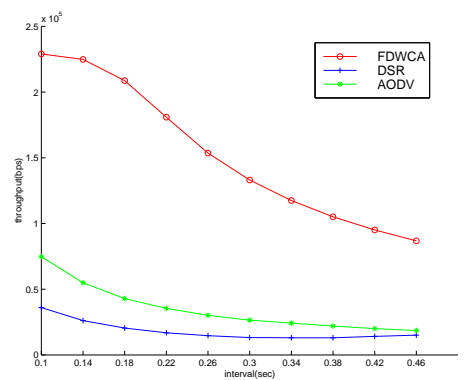

(b) Network throughput
, Fig. 8. Packet delivery ratio and network throughput per round, when a 40 -nodes network varies reporting interval in range of $[0.10,0.46]$ seconds.

To evaluate packet delivery ratio, we fix the network size at 40 and vary the reporting interval from 0.10 to 0.46 seconds. The comparison of three methods is shown in Figure 8(a). As the reporting interval increases (or reporting frequency decreases), the packet delivery ratio in FDWCA algorithm reaches 100 percent after reporting interval increases to around 0.22 seconds, while in DSR and AODV it reaches 100 percent fairly slow. Our simulations (figure not reported here due to space limit) show that only when the network load is very low (the node's reporting interval is around 2 seconds), AODV and DSR start to have $100 \%$ delivery ratio. In many applications, one hundred percent delivery ratio need be guaranteed. That is to say, FDWCA can enable finer scale data collection (e.g., more frequent data sampling is possible) than DSR and AODV. Figure 8(b) shows that our scheduling method FDWCA achieves much better throughput than both AODV and DSR. Notice that for reporting interval of 0.1 seconds with each reporting packet of size 128 Bytes, the total traffic demand produced by all $n=40$ nodes is $\frac{1}{0.1} \cdot n \cdot 1 \mathrm{Kbps}=$ 400 Kbps. The delivery ratio is about $58 \%$, which matches the observed throughput at about 230Kbps in Figure 8(b). 
The above simulation is based on the 802.11 networks, where MAC protocol is a variation of CSMA/CA. Our scheduling algorithm may work better if a TDMA-like MAC protocol is used. Overall, the simulation shows the overwhelming advantage of proper link scheduling: it not only increases network throughput, but also reduces the energy consumption.

\section{RELATED WORK}

Scheduling has been studied extensively in the past few years due to its application for assigning time slots in TDMA MAC protocols. Scheduling can be reduced to different coloring problems: edge coloring and vertex coloring.

Edge coloring, in which every edge corresponds to a valid communication link, is a natural way to capture the link scheduling problem. An edge coloring is valid if no two incident edges share the same color. Vizing's theorem [26] states that a valid edge coloring for an indirected graph can be obtained by using at most $\Delta+1$ colors, where $\Delta$ is the maximum node degree in the graph. On the other hand, any edge coloring needs at least $\Delta$ colors. Any edge coloring that uses $\Theta(\Delta)$ colors is close to the optimal. Panconesi and Srinivasan [27] proposed a randomized distributed edge coloring method that uses at most $2 \Delta+1$ colors. To some extent, this captures some transmission restrictions in ad hoc and sensor network in which no node can receive or send at the same time slot, but it did not address some other interferences such as secondary interference. When one has a valid edge coloring, it can be easily mapped to a TDMA scheduling. However, it is possible that two communication links sharing the same color still interfere with each other in a wireless network. In order to remedy this, Gandham et al. [28] proposed to use a two phase scheduling method: in the 1st phase, a distributed valid edge coloring is obtained; in the 2 nd phase, a valid scheduling taken into account the secondary interference is obtained. In essence, [28] is based on the protocol interference model. The overall scheduling in [28] only provided a performance guarantee when the conflicting links form a tree. Jain et al. [13] proposed a new concept conflict graph that captures the interference in a wireless network.

Vertex coloring is one of the most fundamental NP-hard problems in graph theory and has been thoroughly studied. A vertex coloring is valid iff any two adjacent vertices receive different colors. The minimum number that is needed for a valid vertex coloring for a graph $G$ is known as the chromatic number $\chi(G)$. It is known that for general graph, the chromatic number cannot be approximated within $n^{1-\varepsilon}$ for any $\varepsilon>0$, unless ZPP=NP [29]. For vertex coloring of a general graph $G$, it was proved that, every graph $G$ can be colored using $\delta(G)+1$ colors. Then Hochbaum [19] presented a method to find the value of $\delta(G)$ and color $G$ using $\delta(G)+1$ colors in $O(|V|+|E|)$ time. Ramanathan [9] proposed a unified framework for TDMA, FDMA and CDMA based multi-hop wireless networks. They also proposed a timeslot assignment to edges; the number of timeslots required is at most $O(\theta)$ times the optimum, where $\theta$ is the thickness of a graph, i.e., the minimum number of planar graphs into which the network can be decomposed. Krumke et al. [5] proposed efficient approximation algorithms for the distance- 2 vertex coloring problem for various geometric graphs including $(r, s)$-civilized graphs, planar graphs, graphs with bounded genus, etc. In [6], Kumar et al. studied packet-scheduling under RTS/CTS interference model and gave polylogarithmic/constant factor approximation algorithms for various families of disk graphs and randomized near-optimal approximation algorithms for general graphs.
Several distributed algorithms that use $O(\Delta)$ colors have been proposed in literatures. A $(\Delta+1)$-coloring can be computed in time $O(\log n+\Delta)$ [30] or $O(\Delta \log n)$ [31]. In [20], Maraco et al. proposed a distributed algorithm that computed an $O(\Delta)$ coloring in time $O(\log n)$. All of the above distributed algorithms do not take the interference into account and is based on the message passing model, which implies that the actual time used in a wireless environment could be much larger [8]. Recently, Moscibroda et al. [8] proposed an $O(\Delta)$ distributed coloring method with time-complexity $O(\Delta \log n)$. It is worth to point out that the coloring in [8] considered a simple interference model and the time is close to time needed in practice. However, the coloring in [8] is based on the assumption that the wireless ad hoc network can be modeled as a unit disk graph (UDG), i.e., their method will return a coloring that only guarantees that any nodes that are adjacent in the UDG will get different colors; nodes that are not adjacent in UDG may get the same color. In addition, they assumed that all nodes have the same transmission range and same interference range as its transmission range. This is different from the interference-free scheduling studied in this paper.

Kodialam and Nandagopal [2] studied the effect of interference on the achievable rate region in multi-hop wireless networks. They treated the interference models as linear constraints and solve the flow problem using linear program. In [3], the same authors considered the problem of jointly routing the flows and scheduling transmissions to achieve a given rate vector using the protocol model of interference. They developed necessary and sufficient conditions for the achievable rate vector. They formulated the problem as a linear programming problem and implemented primal-dual algorithms for solving the problem. The scheduling problem is solved as a graph edge-coloring problem using existing greedy algorithms. In [4], they extended their work to the multi-radio multi-channel wireless mesh networks.

Kumar et al. [7] developed analytical performance evaluation models and distributed algorithms for routing and scheduling which incorporate fairness, energy and dilation (path-length) requirements and provide a unified framework for utilizing the network close to its maximum throughput capacity. Alicherry et al. [1] mathematically formulated the joint channel assignment and routing problem in multi-radio mesh networks, and established necessary and sufficient conditions under which interference free link communication schedule can be obtained and designed an simple greedy algorithm to compute such a schedule. Notice that the studied network in [1] is restricted to be a UDG, i.e., the uniform interference range is assumed to be a fixed multiple of the uniform communication range.

Recently, Chen et al. [32], [33] also studied the cross-layer optimization of congestion control and routing together with scheduling problem under interference.

\section{CONCLUSION}

In this paper, we considered the problem of max-throughput (or max-fairness) routing and an interference-aware link scheduling for a wireless network. We assumed a general model for wireless networks, i.e., nodes could have different transmission ranges and different interference ranges, and a link $u v$ may not exist even if $\|u v\|$ is less than the transmission range of node $u$. We presented a linear programming formulation to find a flow routing whose achieved throughput (or fairness) is at least a constant fraction of the optimum and then used the link coloring to resolve the scheduling problem. We presented both centralized 
and distributed scheduling algorithms that use time-slots within a constant factor of the optimum. We also pointed out that the simple link coloring does not imply a good throughput, and then proposed efficient algorithms for general weighted link coloring, which can obtain link scheduling with proven performances. We conducted extensive simulations for our scheduling algorithms. Our theoretical results are corroborated by our simulation studies.

Challenges and Future Work: There are still a number of challenging questions left for future research. The first question is how to efficiently collect the information about the interfering links of a given link. This is not an issue in the previous studies since they assumed a UDG model and the same interference range for all nodes. However, when the interference range is larger than the transmission range, the information on the links within the interference area of a receiver can not be directly collected, since these links may be outside the transmission range. Clearly, collection can only be done with helps of relaying from other nearby nodes. By assuming a fixed interference range and position information available at each node, this process can be done by collecting multihop neighborhood information. However, due to blocking or fading, fixed interference range maybe inaccurate or not practical. The second question is how to improve the overall time complexity of our distributed algorithms. The results presented in [8] may give some insights on this but it is not obvious because the model used here is more complicated than the model used in [8]. We suspect the existence of poly-logarithmic time distributed algorithms for problems studied in this paper under the unstructured environment [8]. The third question is how to solve joint routing and scheduling problem when the link capacity is not fixed. Note that here we assume that the link capacity $c(e)$ is fixed. However, it has been observed that in an interference-limited wireless network, data rates attainable in each link are not fixed and can be a function of SINR at a receiver of the link. In other words, the link capacity depends on the transmission activity of nodes around the receiver. It becomes more challenging to design efficient routing and scheduling method under such link capacity model. Recently, researchers [34]-[36] began to study similar joint-optimization problems under the new characteristics of the link capacity. The forth question is to study the link scheduling in an asynchronized environment. We believe that our methods still apply with small modifications. The last but not the least problem is to study the link scheduling in a dynamic environment where the traffic load on links could change dynamically.

\section{ACKNOWLEDGMENT}

The work was supported in part by the US National Science Foundation (NSF) under Grant No. CNS-0721666 and CCR0311174 and by US National Aeronautics and Space Administration (NASA) under Grant No. ESTO 05-AIST05-0082.

\section{REFERENCES}

[1] M. Alicherry, R. Bhatia, and L. Li. Joint channel assignment and routing for throughput optimization in multi-radio wireless mesh networks. In Proc. of ACM MobiCom, 2005.

[2] M. Kodialam and T. Nandagopal. The effect of interference on the capacity of multi-hop wireless networks. In Proc. of IEEE ISIT, 2004.

[3] M. Kodialam and T. Nandagopal. Characterizing achievable rates in multi-hop wireless networks: the joint routing and scheduling problem. In Proc. of ACM MobiCom, 2003.

[4] M. Kodialam and T. Nandagopal. Characterizing the capacity region in multi-radio multi-channel wireless mesh networks. In Proc. of ACM MobiCom, 2005.

[5] S. Krumke, M. Marathe, and S.S. Ravi. Models and approximation algorithms for channel assignment in radio networks. ACM Wireless Networks, 7(6):575-584, 2001.
[6] A. Kumar, M. Marathe, S. Parthasarathy, and A. Srinivasan. End-to-end packet-scheduling in wireless ad-hoc networks. In Proc. of ACM SODA, 2004.

[7] V.S.A. Kumar, M.V. Marathe, S. Parthasarathy, and A. Srinivasan. Algorithmic aspects of capacity in wireless networks. SIGMETRICS Perform. Eval. Rev., 33(1):133-144, 2005.

[8] T. Moscibroda and R. Wattenhofer. Coloring unstructured radio networks. In Proc. of ACM SPAA, 2005.

[9] S. Ramanathan. A unified framework and algorithm for channel assignment in wireless networks. Wireless Net., 5(2):81-94, 1999.

[10] A. Ephremedis and T. Truong. Scheduling broadcasts in multihop radio networks. IEEE Tran. on Communications, 38(4):456-460, 1990.

[11] E. Arikan. Some complexity results about packet radio networks. IEEE Tran. on Information Theory, 30(4):190-198, 1984.

[12] S. Even, O. Goldreich, S. Moran, and P. Tong. On the NP completeness of certain network testing problems. Networks, 14(1):1-24 1984.

[13] K. Jain, J. Padhye, V.N. Padmanabhan, and L. Qiu. Impact of interference on multi-hop wireless network performance. In Proc. of ACM MobiCom, 2003.

[14] W. Wang, Y. Wang, X.-Y. Li, W.Z. Song, and O. Frieder. Efficient Interference-Aware TDMA Link Scheduling for Static Wireless Networks, In Proc. of ACM MobiCom, 2006.

[15] P. Gupta and P. Kumar. Capacity of wireless networks. IEEE Tran. on Information Theory, IT-46(2):388-404, 2000.

[16] J. Gronkvist and A. Hansson. Comparison between graph-based and interference-based STDMA scheduling. In Proc. of ACM MobiHoc, 2001.

[17] A. Behzad and I. Rubin. On the performance of graph-based scheduling algorithms for packet radio networks. In Proc. of IEEE GlobeCom, 2003.

[18] S. Yi, Y. Pei, S. Kalyanaraman. On the capacity improvement of ad hoc wireless networks using directional antennas. In Proc. of ACM MobiHoc, 2003.

[19] D.S. Hochbaum. Efficient bounds for the stable set, vertex cover, and set packing problems. Discrete Applied Math., 6:243-254, 1983.

[20] G.D. Marco and A. Pelc. Fast distributed graph coloring with $o(\Delta)$ colors. In Proc. of ACM SODA, 2001.

[21] S. Irani, Coloring inductive graphs on-line, Algorithmica, 11(1):53-72, 1994

[22] I. Caragiannis, A.V. Fishkin, C. Kaklamanis, and E. Papaioannou. A Tight Bound for Online Colouring of Disk Graphs. Theoretical Computer Science, 384(2-3):152-160, 2007

[23] QualNet Simulator. http://www.scalable-networks.com

[24] David B Johnson and David A Maltz. Dynamic Source Routing in Ad Hoc Wireless Networks. In Mobile Computing, Kluwer Academic Publishers, 1996.

[25] C. E. Perkins and E. Belding-Royer, and S. Das. Ad-hoc On-Demand Distance Vector Routing. IETF RFC 3561, 2003.

[26] C. Berge. Graphs and Hyper Graphs. North-Holland, 1973.

[27] A. Panconesi and A. Srinivasan. Improved distributed algorithms for coloring and network decomposition problems. In Proc. of ACM STOC, 1992.

[28] S. Gandham, M. Dawande, and R. Prakash. Link scheduling in sensor networks: Distributed edge coloring revisited. In Proc. of IEEE InfoCom, 2005.

[29] U. Feige and J. Kilian. Zero knowledge and the chromatic number. Journal of Computer System Science, 57(2):187-199, 1998.

[30] A. Panconesi and R. Rizzi. Some simple distributed algorithms for sparse networks. Distributed Computing, 14(2):97-100, 2001.

[31] A. Goldberg, S. Plotkin, and G. Shannon. Parallel symmetry breaking in sparse graphs. In Proc. of ACM STOC, 1987.

[32] L. Chen, S.H. Low, and J.C. Doyle. Joint congestion control and media access control design for wireless ad hoc networks. In Proc. of IEEE InfoCom, 2005.

[33] L. Chen, S.H. Low, M. Chiang, and J.C. Doyle. Cross-layer congestion control, routing and scheduling design in ad hoc wireless networks. In Proc. of IEEE InfoCom, 2006.

[34] M. J. Neely, E. Modiano, and C. E. Rohrs. Dynamic power allocation and routing time varying wireless networks. IEEE J. Sel. Areas Comm., 23(1):89-103, 2005.

[35] L. Xiao, M. Johansson and S. Boyd. Simultaneous routing and resource allocation via dual decomposition. IEEE Trans. Comm., 52(7):11361144, 2004.

[36] L. Bui, A. Eryilmaz, R. Srikant, and X. Wu. Joint asynchronous congestion control and distributed scheduling for multi-hop wireless networks. In Proc. of IEEE INFOCOM, 2006. 